\title{
Periodic boundary value problems for two classes of nonlinear fractional differential equations
}

\author{
Wenjuan Yao ${ }^{1}$, Zhichang Guo ${ }^{1}$ and Jiebao Sun ${ }^{1 *}$ (D)
}

\section{"Correspondence:}

sunjiebao@hit.edu.cn

1 Department of Mathematics, Harbin Institute of Technology,

Harbin, China

\begin{abstract}
By using the coincidence degree theorem, we obtain a new result on the existence
solutions for a class of fractional differential equations with periodic boundary value
conditions, where a certain nonlinear growth condition of the nonlinearity needs
be satisfied. Furthermore, we study another class of differential equations of fraction
order with periodic boundary conditions at resonance. A new result on the existenc
of positive solutions is presented by use of a Leggett-Williams norm-type theorem
coincidences. Two examples are given to illustrate the main result at the end of this
paper.
Keywords: Fractional differential equations; Periodic boundary value problem;
Existence; Coincidence degree; Positive solution
Abstract
By using the coincidence degree theorem, we obtain a new result on the existence of
solutions for a class of fractional differential equations with periodic boundary value
conditions, where a certain nonlinear growth condition of the nonlinearity needs to
be satisfied. Furthermore, we study another class of differential equations of fractional
order with periodic boundary conditions at resonance. A new result on the existence
of positive solutions is presented by use of a Leggett-Williams norm-type theorem for
coincidences. Two examples are given to illustrate the main result at the end of this
paper.
Keywords: Fractional differential equations; Periodic boundary value problem;
Existence; Coincidence degree; Positive solution

Abstract
By using the coincidence degree theorem, we obtain a new result on the existence of
solutions for a class of fractional differential equations with periodic boundary value
conditions, where a certain nonlinear growth condition of the nonlinearity needs to
be satisfied. Furthermore, we study another class of differential equations of fractiona
order with periodic boundary conditions at resonance. A new result on the existence
of positive solutions is presented by use of a Leggett-Williams norm-type theorem for
coincidences. Two examples are given to illustrate the main result at the end of this
paper.
Keywords: Fractional differential equations; Periodic boundary value problem;
Existence; Coincidence degree; Positive solution

Abstract
By using the coincidence degree theorem, we obtain a new result on the existence of
solutions for a class of fractional differential equations with periodic boundary value
conditions, where a certain nonlinear growth condition of the nonlinearity needs to
be satisfied. Furthermore, we study another class of differential equations of fractiona
order with periodic boundary conditions at resonance. A new result on the existence
of positive solutions is presented by use of a Leggett-Williams norm-type theorem for
coincidences. Two examples are given to illustrate the main result at the end of this
paper.
Keywords: Fractional differential equations; Periodic boundary value problem;
Existence; Coincidence degree; Positive solution

Abstract
By using the coincidence degree theorem, we obtain a new result on the existence of
solutions for a class of fractional differential equations with periodic boundary value
conditions, where a certain nonlinear growth condition of the nonlinearity needs to
be satisfied. Furthermore, we study another class of differential equations of fractional
order with periodic boundary conditions at resonance. A new result on the existence
of positive solutions is presented by use of a Leggett-Williams norm-type theorem for
coincidences. Two examples are given to illustrate the main result at the end of this
paper.
Keywords: Fractional differential equations; Periodic boundary value problem;
Existence; Coincidence degree; Positive solution

Abstract
By using the coincidence degree theorem, we obtain a new result on the existence of
solutions for a class of fractional differential equations with periodic boundary value
conditions, where a certain nonlinear growth condition of the nonlinearity needs to
be satisfied. Furthermore, we study another class of differential equations of fractional
order with periodic boundary conditions at resonance. A new result on the existence
of positive solutions is presented by use of a Leggett-Williams norm-type theorem for
coincidences. Two examples are given to illustrate the main result at the end of this
paper.
Keywords: Fractional differential equations; Periodic boundary value problem;
Existence; Coincidence degree; Positive solution

Abstract
By using the coincidence degree theorem, we obtain a new result on the existence of
solutions for a class of fractional differential equations with periodic boundary value
conditions, where a certain nonlinear growth condition of the nonlinearity needs to
be satisfied. Furthermore, we study another class of differential equations of fractional
order with periodic boundary conditions at resonance. A new result on the existence
of positive solutions is presented by use of a Leggett-Williams norm-type theorem for
coincidences. Two examples are given to illustrate the main result at the end of this
paper.
Keywords: Fractional differential equations; Periodic boundary value problem;
Existence; Coincidence degree; Positive solution

Abstract
By using the coincidence degree theorem, we obtain a new result on the existence
solutions for a class of fractional differential equations with periodic boundary value
conditions, where a certain nonlinear growth condition of the nonlinearity needs to
be satisfied. Furthermore, we study another class of differential equations of fractional
order with periodic boundary conditions at resonance. A new result on the existence
of positive solutions is presented by use of a Leggett-Williams norm-type theorem for
coincidences. Two examples are given to illustrate the main result at the end of this
paper.
Keywords: Fractional differential equations; Periodic boundary value problem;
Existence; Coincidence degree; Positive solution

Abstract
By using the coincidence degree theorem, we obtain a new result on the existence
solutions for a class of fractional differential equations with periodic boundary value
conditions, where a certain nonlinear growth condition of the nonlinearity needs
be satisfied. Furthermore, we study another class of differential equations of fraction
order with periodic boundary conditions at resonance. A new result on the existenc
of positive solutions is presented by use of a Leggett-Williams norm-type theorem
coincidences. Two examples are given to illustrate the main result at the end of this
paper.
Keywords: Fractional differential equations; Periodic boundary value problem;
Existence; Coincidence degree; Positive solution

Abstract
By using the coincidence degree theorem, we obtain a new result on the existence
solutions for a class of fractional differential equations with periodic boundary value
conditions, where a certain nonlinear growth condition of the nonlinearity needs
be satisfied. Furthermore, we study another class of differential equations of fraction
order with periodic boundary conditions at resonance. A new result on the existenc
of positive solutions is presented by use of a Leggett-Williams norm-type theorem
coincidences. Two examples are given to illustrate the main result at the end of this
paper.
Keywords: Fractional differential equations; Periodic boundary value problem;
Existence; Coincidence degree; Positive solution

Abstract
By using the coincidence degree theorem, we obtain a new result on the existence
solutions for a class of fractional differential equations with periodic boundary value
conditions, where a certain nonlinear growth condition of the nonlinearity needs
be satisfied. Furthermore, we study another class of differential equations of fraction
order with periodic boundary conditions at resonance. A new result on the existenc
of positive solutions is presented by use of a Leggett-Williams norm-type theorem
coincidences. Two examples are given to illustrate the main result at the end of this
paper.
Keywords: Fractional differential equations; Periodic boundary value problem;
Existence; Coincidence degree; Positive solution
\end{abstract}

\section{Introduction}

Fractional calculus is the emerging mathematical field which is devoted to studying convolution-type pseudo-differential operators, specifically integrals and derivatives of any arbitrary real or complex order. In recent years, the fractional calculus has been considered as the best tool for the generalization of fractional differential equations. It has become more and more important in many fields of science and engineering, such as chemistry, biology, electricity, control theory, and image processing (see [1-4]). In addition, a considerable amount of progress has recently been made in the study of fractional calculus, and a number of results on this subject have been now achieved. For readers new to this subject, we cite a few proper ones of the books, and a comprehensive treatment of this subject and its applications can be found in [5-8].

In the past few decades, boundary value problems of fractional order involving a variety of boundary conditions have been studied by several researchers. We refer the readers to [9-15] and the references cited therein. Moreover,the existence of solutions to the fractional differential equations with anti-periodic boundary value conditions has been studied by many authors (see [16-21]). But the periodic boundary value problems for nonlinear fractional differential equations are seldom considered. Recently, the existence of solutions to nonlinear integer order periodic boundary value problems has been discussed in many articles (see [22-25]). Here, we point out that a few authors have recently considered fractional problems. In these formulations, the first order derivatives are replaced 
by fractional derivatives, which causes many difficulties in solving the resulting problems. In [26], Chen and Liu investigated the existence of solutions for the following periodic boundary value problem:

$$
\left\{\begin{array}{l}
x^{\prime \prime}(t)=f\left(t, x(t), D_{0^{+}}^{\alpha} x(t)\right), \quad t \in[0,1] \\
x(0)=x(1), \quad D_{0^{+}}^{\alpha} x(0)=D_{0^{+}}^{\alpha} x(1)
\end{array}\right.
$$

where $0<\alpha<2$ is a real number, $D_{0+}^{\alpha}$ is a Caputo fractional derivative, and $f:[0,1] \times \mathbb{R}^{2} \rightarrow$ $\mathbb{R}$ is continuous.

In [27], Hu and Zhang gained the existence of positive solutions of fractional differential equation with periodic boundary value conditions of the form:

$$
\left\{\begin{array}{l}
D_{0^{+}}^{\alpha} u(t)=f(t, u(t)), \quad t \in[0,1], \\
u(0)=u(1), \quad u^{\prime}(0)=u^{\prime}(1), \quad u^{\prime \prime}(0)=u^{\prime \prime}(1)
\end{array}\right.
$$

where $2<\alpha<3$ is a real number, $D_{0_{+}}^{\alpha}$ is a Caputo fractional derivative, and $f:[0,1] \times \mathbb{R} \rightarrow$ $\mathbb{R}$ is continuous.

Motivated by the work mentioned previously, this paper investigates the existence of solutions for two kinds of periodic boundary value problems (PBVP for short) of nonlinear fractional differential equations. The first one is described in the following form:

$$
\left\{\begin{array}{l}
D_{0^{+}}^{\beta}\left(p(t) D_{0^{+}}^{\alpha} x(t)\right)=f\left(t, x(t), D_{0^{+}}^{\alpha} x(t)\right), \quad t \in[0, T], \\
x(0)=x(T), \quad D_{0^{+}}^{\alpha} x(0)=D_{0^{+}}^{\alpha} x(T),
\end{array}\right.
$$

where $0<\alpha, \beta \leq 1, D_{0^{+}}^{\alpha}$ is the Caputo fractional derivative, $f:[0, T] \times \mathbb{R}^{2} \rightarrow \mathbb{R}$ is continuous, $p(t) \in C^{1}[0, T], p(0)=p(T)$, and there exists a positive constant $M$ such that $p(t) \geq M$ for all $t \in[0, T]$.

However, the PBVP

$$
\left\{\begin{array}{l}
D_{0^{+}}^{\beta}\left(p(t) D_{0^{+}}^{\alpha} x(t)\right)=h(t), \quad t \in[0, T], \\
x(0)=x(T), \quad D_{0^{+}}^{\alpha} x(0)=D_{0^{+}}^{\alpha} x(T),
\end{array}\right.
$$

is not solvable for each $h \in C([0, T], \mathbb{R})$, and, when solvable, has no unique solution because $x(t)+c, \forall c \in \mathbb{R}$ is a solution together with $x(t)$. In this case, a trivial necessary condition for the solvability of PBVP (2) is that

$$
\bar{h}=\frac{\beta}{T^{\beta}} \int_{0}^{T}(T-s)^{\beta-1} h(s) d s=0 .
$$

Furthermore, we change the range of $\alpha$ and take $p(t)=1$, i.e., consider the following PBVP:

$$
\begin{cases}D_{0^{+}}^{\beta} D_{0^{+}}^{\alpha} x(t)=g\left(t, x(t), D_{0^{+}}^{\alpha} x(t)\right), & t \in[0, T], \\ x(0)=x(T), \quad x^{\prime}(0)=x^{\prime}(T), & D_{0^{+}}^{\alpha} x(0)=D_{0^{+}}^{\alpha} x(T),\end{cases}
$$

where $0<\beta \leq 1,1<\alpha \leq 2, \alpha+\beta \geq 2, g:[0, T] \times \mathbb{R}^{2} \rightarrow \mathbb{R}$ is continuous. Then the existence of solutions for this PBVP is obtained under some assumptions of function $g$. 
This paper is organized as follows. In Sect. 2, we establish an existence theorem of solutions for PBVP (1) under nonlinear growth restriction of $f$. The key is an analytic technique from the theory of coincidence degree. In Sect. 3, we obtain the existence of positive solutions of (3) by Theorem 3. Two illustrative examples of nonlinear fractional problems with periodic boundary conditions are shown in Sect. 4.

\section{Existence for PBVP (1)}

\subsection{Preliminaries}

In this subsection, as preliminaries, we firstly present some basic definitions and formulations on fractional calculus. For further background knowledge of fractional calculus, we refer the readers to [6].

Definition 1 The Riemann-Liouville fractional integral operator of order $\alpha>0$ of a function $u:(0,+\infty) \rightarrow \mathbb{R}$ is given by

$$
I_{0^{+}}^{\alpha} u(t)=\frac{1}{\Gamma(\alpha)} \int_{0}^{t}(t-s)^{\alpha-1} u(s) d s,
$$

provided that the right-hand side integral is pointwise defined on $(0,+\infty)$.

Definition 2 The Caputo fractional derivative of order $\alpha>0$ of a function $u:(0,+\infty) \rightarrow \mathbb{R}$ is given by

$$
D_{0^{+}}^{\alpha} u(t)=I_{0^{+}}^{n-\alpha} \frac{d^{n} u(t)}{d t^{n}}=\frac{1}{\Gamma(n-\alpha)} \int_{0}^{t}(t-s)^{n-\alpha-1} u^{(n)}(s) d s,
$$

where $n$ is the smallest integer greater than or equal to $\alpha$, provided that the right-hand side integral is pointwise defined on $(0,+\infty)$.

Lemma 1 ([7]) The fractional differential equation $D_{0+}^{\alpha} y(t)=0$ has solution $y(t)=c_{0}+c_{1} t+$ $\cdots+c_{n-1} t^{n-1}, c_{i} \in \mathbb{R}, i=0,1, \ldots, n-1, n=[\alpha]+1$. Furthermore, for $y \in A C^{n}[0, T]$,

$$
\left(I_{0+}^{\alpha} D_{0+}^{\alpha} y\right)(t)=y(t)-\sum_{k=0}^{n-1} \frac{y^{(k)}(0)}{k !} t^{k}
$$

and

$$
\left(D_{0+}^{\alpha} I_{0+}^{\alpha} y\right)(t)=y(t)
$$

Lemma 2 ([7]) The relation

$$
I_{a+}^{\alpha} I_{a+}^{\beta} f(x)=I_{a+}^{\alpha+\beta} f(x)
$$

is valid in the following case: $\beta>0, \alpha+\beta>0, f(x) \in L_{1}(a, b)$.

Lemma 3 ([28]) Let $X, Y$ be real Banach spaces, $L: \operatorname{dom} L \subset X \rightarrow Y$ be a Fredholm operator with index zero, and $P: X \rightarrow X, Q: Y \rightarrow Y$ be projectors such that

$$
\operatorname{Im} P=\operatorname{ker} L, \quad \operatorname{ker} Q=\operatorname{Im} L, \quad X=\operatorname{ker} L \oplus \operatorname{ker} P, \quad Y=\operatorname{Im} L \oplus \operatorname{Im} Q
$$

It follows that $\left.L\right|_{\operatorname{dom} L \cap \operatorname{ker} P}: \operatorname{dom} L \cap \operatorname{ker} P \rightarrow \operatorname{Im} L$ is invertible. 
Denote $Y=C([0, T], \mathbb{R})$ with the norm $\|y\|_{\infty}=\max _{t \in[0, T]}|y(t)|, X=\left\{x \mid x, D_{0^{+}}^{\alpha} x \in Y\right\}$ and

$$
X_{T}=\left\{x \in X \mid x(0)=x(T), D_{0^{+}}^{\alpha} x(0)=D_{0^{+}}^{\alpha} x(T)\right\}
$$

with the norm $\|x\|_{X}=\max \left\{\|x\|_{\infty},\left\|D_{0^{+}}^{\alpha} x\right\|_{\infty}\right\}$. It is easy to see that $X$ and $X_{T}$ are Banach spaces.

Define an operator $L: \operatorname{dom} L \subset X \rightarrow Y$ by

$$
L x=D_{0^{+}}^{\beta}\left(p(t) D_{0^{+}}^{\alpha} x\right),
$$

where

$$
\operatorname{dom} L=\left\{x \in X_{T} \mid D_{0^{+}}^{\beta}\left(p(t) D_{0^{+}}^{\alpha} x\right) \in Y\right\} .
$$

Let $N_{f}: X \rightarrow Y$ be the Nemytskii operator

$$
N_{f} x(t)=f\left(t, x(t), D_{0^{+}}^{\alpha} x(t)\right), \quad \forall t \in[0, T] .
$$

Then PBVP (1) is equivalent to the operator equation

$$
L x=N_{f} x, \quad x \in \operatorname{dom} L .
$$

\subsection{Main result}

In this subsection, by using the coincidence degree theorem, we establish a new existence result on PBVP (1) for the nonlinear fractional differential equation under the nonlinear growth restriction of $f$.

First, we show some lemmas which will play important roles in the proof of the main result.

Consider PBVP (2) with $h \in Y$ such that $\bar{h}=0$, and let $x$ be a solution of PBVP (2). From Lemma 1, we have

$$
p(t) D_{0^{+}}^{\alpha} x(t)=a+I_{0^{+}}^{\beta} h(t)=a+\frac{1}{\Gamma(\beta)} \int_{0}^{t}(t-s)^{\beta-1} h(s) d s, \quad \forall a \in \mathbb{R},
$$

which together with the periodic boundary condition $x(0)=x(T)$ implies that

$$
\int_{0}^{T}(T-s)^{\alpha-1}\left\{\frac{a+I_{0^{+}}^{\beta} h(s)}{p(s)}\right\} d s=0
$$

For any fixed $l \in Y$, define the function $G_{l}(a): \mathbb{R} \rightarrow \mathbb{R}$ by

$$
G_{l}(a)=\frac{\alpha}{T^{\alpha}} \int_{0}^{T}(T-s)^{\alpha-1}\left\{\frac{a+l(s)}{p(s)}\right\} d s .
$$

Then we have the following lemma. 
Lemma 4 The function $G_{l}(a)$ has the following properties:

(i) for any fixed $l \in Y$, the equation

$$
G_{l}(a)=0
$$

has a unique solution $\tilde{a}(l)$;

(ii) the function $\tilde{a}: Y \rightarrow \mathbb{R}$, defined in (1), is continuous and sends bounded sets into bounded sets.

Proof (i) By (7), we have

$$
\left(G_{l}\left(a_{1}\right)-G_{l}\left(a_{2}\right)\right)\left(a_{1}-a_{2}\right)>0 \text { for } a_{1} \neq a_{2},
$$

hence the solution of (8) is unique. To prove the existence, we will show that $C_{l}(a) \cdot a>0$ for $|a|$ sufficiently large. Since

$$
\begin{aligned}
G_{l} a \cdot a= & \frac{\alpha}{T^{\alpha}} \int_{0}^{T}(T-s)^{\alpha-1} \cdot \frac{a+l(s)}{p(s)} \cdot a d s \\
= & \frac{\alpha}{T^{\alpha}} \int_{0}^{T}(T-s)^{\alpha-1} \cdot \frac{a+l(s)}{p(s)} \cdot(a+l(s)) d s \\
& -\frac{\alpha}{T^{\alpha}} \int_{0}^{T}(T-s)^{\alpha-1} \cdot \frac{a+l(s)}{p(s)} \cdot l(s) d s,
\end{aligned}
$$

then we have

$$
\begin{aligned}
G_{l} a \cdot a \geq & \frac{\alpha}{T^{\alpha}} \int_{0}^{T}(T-s)^{\alpha-1} \cdot \frac{a+l(s)}{p(s)} \cdot(a+l(s)) d s \\
& -\|l\|_{\infty} \frac{\alpha}{T^{\alpha}} \int_{0}^{T}(T-s)^{\alpha-1} \cdot\left|\frac{a+l(s)}{p(s)}\right| d s .
\end{aligned}
$$

From the property of $p(t)$, we have

$$
y \cdot \frac{y}{p(s)} \geq M\left|\frac{y}{p(s)}\right|\left|\frac{y}{p(s)}\right|
$$

for any $y \in \mathbb{R}$. Thus, from (9) and (10), we obtain

$$
G_{l} a \cdot a \geq \frac{\alpha}{T^{\alpha}} \int_{0}^{T}(T-s)^{\alpha-1}\left(M \cdot \frac{a+l(s)}{p(s)}-\|l\|_{\infty}\right)\left|\frac{a+l(s)}{p(s)}\right| d s .
$$

Since $|a| \rightarrow \infty$ implies that $\left|\frac{a+l(t)}{p(t)}\right| \rightarrow \infty$ uniformly for $t \in[0, T]$, we find from (11) that there exists $r>0$ such that

$$
G_{l} a \cdot a>0
$$

for all $a \in \mathbb{R}$ with $|a|=r$. By an elementary topological degree argument, it follows that the equation $G_{l}(a)=0$ has a solution for each $l \in Y$, which by our previous argument is 
unique. In this way, for any $l \in Y$, we define a function $\tilde{a}: Y \rightarrow \mathbb{R}$ which satisfies

$$
\int_{0}^{T}(T-s)^{\alpha-1}\left(\frac{\tilde{a}+l(s)}{p(s)}\right) d s=0 .
$$

To prove (ii), let $\Lambda$ be a bounded subset of $Y$ and $l \in \Lambda$. Then, from (12), we have

$$
\int_{0}^{T}(T-s)^{\alpha-1}\left(\frac{\tilde{a}(l)+l(s)}{p(s)}\right) \tilde{a}(l) d s=0,
$$

and hence

$$
\begin{gathered}
\int_{0}^{T}(T-s)^{\alpha-1}\left(\frac{\tilde{a}(l)+l(s)}{p(s)}\right)(\tilde{a}(l)+l(s)) d s \\
=\int_{0}^{T}(T-s)^{\alpha-1}\left(\frac{\tilde{a}(l)+l(s)}{p(s)}\right) l(s) d s .
\end{gathered}
$$

Suppose that $\{\tilde{a}(l), l \in \Lambda\}$ is not bounded. Then, for an arbitrary $A>0$, there is $l \in \Lambda$ with $\|l\|_{\infty}$ sufficiently large so that

$$
A \leq M\left|\frac{\tilde{a}(l)+l(s)}{p(s)}\right|,
$$

uniformly in $t \in[0, T]$. Hence, by using (10) and (13), we find that

$$
\begin{aligned}
A \int_{0}^{T}(T-s)^{\alpha-1}\left|\frac{\tilde{a}(l)+l(s)}{p(s)}\right| d s & \leq \int_{0}^{T} M(T-s)^{\alpha-1}\left|\frac{\tilde{a}(l)+l(s)}{p(s)}\right|^{2} d s \\
& \leq\|l\|_{\infty} \int_{0}^{T}(T-s)^{\alpha-1}\left|\frac{\tilde{a}(l)+l(s)}{p(s)}\right| d s .
\end{aligned}
$$

Thus $A \leq\|l\|_{\infty}$, which is a contradiction. Therefore $\tilde{a}$ sends bounded sets in $Y$ into bounded sets in $\mathbb{R}$.

Finally, we show the continuity of $\tilde{a}$. Let $\left\{l_{n}\right\}$ be a convergent sequence in $Y$, say $l_{n} \rightarrow l$, as $n \rightarrow \infty$. Since $\left\{a\left(l_{n}\right)\right\}$ is a bounded sequence, any subsequence of it contains a convergent subsequence denoted by $\left\{a\left(l_{n_{j}}\right)\right\}$. Let $a\left(l_{n_{j}}\right)$, as $j \rightarrow \infty$. By letting $j \rightarrow \infty$ in

$$
\int_{0}^{T}(T-s)^{\alpha-1}\left(\frac{\tilde{a}\left(l_{n_{j}}\right)+l_{n_{j}}(s)}{p(s)}\right) d s=0,
$$

we find that

$$
\int_{0}^{T}(T-s)^{\alpha-1}\left(\frac{\hat{a}+l(s)}{p(s)}\right) d s=0,
$$

and hence $\tilde{a}(l)=\hat{a}$, which shows the continuity of $\tilde{a}$.

The proof is complete.

Let $a: Y \rightarrow \mathbb{R}$ be defined by

$$
a(h)=\tilde{a}\left(I_{0^{+}}^{\beta} h\right) .
$$


Then, based on Lemma 4, $a$ is a completely continuous mapping. Furthermore, by (6) and Lemma 1, we obtain that

$$
x(t)=x(0)+I_{0^{+}}^{\alpha}\left(\frac{a(h)+I_{0^{+}}^{\beta} h(t)}{p(t)}\right) .
$$

Lemma 5 Let L be defined by (4), then

$$
\begin{aligned}
& \operatorname{ker} L=\{x \in X \mid x(t)=c, \forall t \in[0, T], c \in \mathbb{R}\}, \\
& \operatorname{Im} L=\left\{y \in Y \mid \int_{0}^{T}(T-s)^{\beta-1} y(s) d s=0\right\} .
\end{aligned}
$$

Proof By Lemma $1, \forall b, c \in \mathbb{R}$, the solution of $D_{0^{+}}^{\beta}\left(p(t) D_{0^{+}}^{\alpha} x(t)\right)=0$ satisfies

$$
x(t)=c+I_{0^{+}}^{\alpha}\left(\frac{b}{p(t)}\right) .
$$

Combining the property of $p(t)$ with periodic boundary value conditions

$$
D_{0^{+}}^{\alpha} x(0)=D_{0^{+}}^{\alpha} x(T) \text { and } x(0)=x(T),
$$

we have $b=0$. That is, (15) holds.

If $y \in \operatorname{Im} L$, then there exists a function $x \in \operatorname{dom} L$ such that $y(t)=D_{0^{+}}^{\beta}\left(p(t) D_{0^{+}}^{\alpha} x(t)\right)$. By Lemma 1, we have

$$
D_{0^{+}}^{\alpha} x(t)=\frac{I_{0^{+}}^{\beta} y(t)+c_{1}}{p(t)}=\frac{\frac{1}{\Gamma(\beta)} \int_{0}^{t}(t-s)^{\beta-1} y(s) d s+c_{1}}{p(t)}, \quad c_{1} \in \mathbb{R} .
$$

From the boundary condition $D_{0^{+}}^{\alpha} x(0)=D_{0^{+}}^{\alpha} x(T)$, it follows that

$$
\int_{0}^{T}(T-s)^{\beta-1} y(s) d s=0
$$

On the other hand, let $y \in Y$ satisfy (17) and

$$
x(t)=I_{0^{+}}^{\alpha}\left(\frac{a(y)+I_{0^{+}}^{\beta} y(t)}{p(t)}\right),
$$

then $D_{0^{+}}^{\alpha} x(0)=D_{0^{+}}^{\alpha} x(T)$. From the definition of mapping $a$, we have

$$
x(0)=0=I_{0^{+}}^{\alpha}\left(\frac{a(y)+I_{0^{+}}^{\beta} y(T)}{p(T)}\right)=x(T) .
$$

Then we have $x \in \operatorname{dom} L$ and $L x(t)=D_{0^{+}}^{\beta}\left(p(t) D_{0^{+}}^{\alpha} x(t)\right)=y(t)$. So $y \in \operatorname{Im} L$. The proof is complete.

Define projectors $P: X \rightarrow X$ and $Q: Y \rightarrow Y$ by

$$
P x(t)=x(0), \quad \forall t \in[0, T],
$$




$$
\begin{aligned}
& Q y(t)=\frac{\beta}{T^{\beta}} \int_{0}^{1}(T-s)^{\beta-1} y(s) d s, \quad \forall t \in[0, T] . \\
& \mathcal{K} h(t)=I_{0^{+}}^{\alpha}\left[\frac{a((I-Q) h)+I_{0^{+}}^{\beta}(I-Q) h(t)}{p(t)}\right], \quad \forall t \in[0, T] .
\end{aligned}
$$

By (14), we can infer that the solution $x \in X_{T}$ of PBVP (2) satisfies the following abstract equation:

$$
x=P x+Q h+\mathcal{K} h .
$$

According to the proof of Lemma 5, we can also infer that the solution $x$ of (20) is also a solution of PBVP (2).

Notice that $a(0)=\tilde{a}(0)=0$, we get $\mathcal{K}(0)=0$.

Lemma 6 The operator $\mathcal{K}$ is a completely continuous operator.

Proof In fact, by the definition of $\mathcal{K}$, it follows that

$$
D_{0^{+}}^{\alpha} \mathcal{K} h(t)=\left[\frac{a((I-Q) h)+I_{0^{+}}^{\beta}(I-Q) h(t)}{p(t)}\right], \quad \forall t \in[0, T] .
$$

Based on the continuity of $Q$, it follows that $\mathcal{K}$ and $D_{0^{+}}^{\alpha} \mathcal{K}$ are continuous in $Y$. That is, $\mathcal{K}$ is a continuous operator.

Let $\Omega \subset Y$ be an arbitrary open bounded set, then $\mathcal{K}(\bar{\Omega})$ and $D_{0^{+}}^{\alpha} \mathcal{K}(\bar{\Omega})$ are bounded. Thus, in view of the Arzelà-Ascoli theorem, it remains to verify that $\mathcal{K}(\bar{\Omega}) \subset X_{T}$ is equicontinuous.

In view of Lemma 4, we deduce that the operator $\left[a((I-Q) h)+I_{0^{+}}^{\beta}(I-Q) h\right]$ is bounded. That is, there exists a positive constant $M_{1}>0$ such that

$$
\left|\left[a((I-Q) h)+I_{0^{+}}^{\beta}(I-Q) h\right](t)\right| \leq M_{1}, \quad \forall h \in \bar{\Omega}, t \in[0, T] .
$$

For $0 \leq t_{1}<t_{2} \leq T, h \in \bar{\Omega}$, we have

$$
\begin{aligned}
\left|\mathcal{K} h\left(t_{2}\right)-\mathcal{K} h\left(t_{1}\right)\right| \\
=\frac{1}{\Gamma(\alpha)} \mid \int_{0}^{t_{2}}\left(t_{2}-s\right)^{\alpha-1} \frac{\left[a((I-Q) h)+I_{0^{+}}^{\beta}(I-Q) h\right]}{p(s)}(s) d s \\
\quad-\int_{0}^{t_{1}}\left(t_{1}-s\right)^{\alpha-1} \frac{\left[a((I-Q) h)+I_{0^{+}}^{\beta}(I-Q) h\right]}{p(s)}(s) d s \mid \\
\leq \frac{M_{1}}{\Gamma(\alpha) M}\left\{\int_{0}^{t_{1}}\left[\left(t_{1}-s\right)^{\alpha-1}-\left(t_{2}-s\right)^{\alpha-1}\right] d s+\int_{t_{1}}^{t_{2}}\left(t_{2}-s\right)^{\alpha-1} d s\right\} \\
=\frac{M_{1}}{\Gamma(\alpha+1) M}\left[t_{1}^{\alpha}-t_{2}^{\alpha}+2\left(t_{2}-t_{1}\right)^{\alpha}\right] .
\end{aligned}
$$

Since $t^{\alpha}$ is uniformly continuous in $[0, T]$, by the definition of $\mathcal{K}$, we can see that $\mathcal{K}(\bar{\Omega}) \subset Y$ is equicontinuous. Likewise, it follows that $\left[a(I-Q)+I_{0^{+}}^{\beta}(I-Q)\right](\bar{\Omega}) \subset Y$ is equicontinuous. This, together with the property of $p(s)$, implies that $D_{0^{+}}^{\alpha} \mathcal{K}(\bar{\Omega}) \subset Y$ is also equicontinuous. Thus we prove that the operator $\mathcal{K}: Y \rightarrow X_{T}$ is compact. The proof is complete. 
Lemma 7 Let $f:[0, T] \times \mathbb{R}^{2} \rightarrow \mathbb{R}$ be continuous, $L, N_{f}$, Q be defined respectively by (4), (5), (18), and $\Omega$ be an open bounded subset of $X_{T}$ such that $\operatorname{dom} L \cap \bar{\Omega} \neq \emptyset$. Assume that the following conditions are satisfied:

$\left(C_{1}\right)$ for each $\lambda \in(0,1)$, the equation

$$
L x=\lambda N_{f} x
$$

has no solution on $(\operatorname{dom} L \backslash \operatorname{ker} L) \cap \partial \Omega$;

$\left(C_{2}\right)$ the equation $Q N_{f} x=0$ has no solution on $\operatorname{ker} L \cap \partial \Omega$;

$\left(C_{3}\right)$ the Brouwer degree $\operatorname{deg}\left(\left.Q N_{f}\right|_{\operatorname{ker} L}, \Omega \cap \operatorname{ker} L, 0\right) \neq 0$.

Then the equation $L x=N_{f} x$ has at least one solution in $\operatorname{dom} L \cap \bar{\Omega}$.

Proof Let us consider the homotopic equation of $L x=N_{f} x$ as follows:

$$
L x=\lambda N_{f} x+(1-\lambda) Q N_{f} x, \quad x \in \operatorname{dom} L .
$$

That is,

$$
\left\{\begin{array}{l}
D_{0^{+}}^{\beta}\left(p(t) D_{0^{+}}^{\alpha} x(t)\right) \\
\quad=\lambda f\left(t, x(t), D_{0^{+}}^{\alpha} x(t)\right)+(1-\lambda) \frac{\beta}{T^{\beta}} \int_{0}^{T}(T-s)^{\beta-1} f\left(s, x(s), D_{0^{+}}^{\alpha} x(s)\right) d s, \\
x(0)=x(T), \quad D_{0^{+}}^{\alpha} x(0)=D_{0^{+}}^{\alpha} x(T) .
\end{array}\right.
$$

Obviously, for $\lambda \in(0,1]$, if $x$ is a solution of Eq. (21) or Eq. (22), then we have

$$
Q N_{f} x(t)=\frac{\beta}{T^{\beta}} \int_{0}^{T}(T-s)^{\beta-1} f\left(s, x(s), D_{0^{+}}^{\alpha} x(s)\right) d s=0 .
$$

It can be seen that Eq. (21) and Eq. (22) have the same solutions. Furthermore, Eq. (22) is equivalent to the following form:

$$
x=G_{f}(x, \lambda),
$$

where $G_{f}: X_{T} \times[0,1] \rightarrow X_{T}$ is defined by

$$
\begin{aligned}
G_{f}(x, \lambda) & =P x+Q N_{f} x+\left[\mathcal{K} \circ\left(\lambda N_{f}(1-\lambda) Q N_{f}\right)\right] x \\
& =P x+Q N_{f} x+\left[\mathcal{K} \circ\left(\lambda(I-Q) N_{f}\right)\right] x .
\end{aligned}
$$

In view of the continuity of $f$ and Lemma 6 , it is known that $G_{f}$ is a completely continuous operator.

For $\lambda=1$, we assume that Eq. (23) does not have a solution on $\partial \Omega$. Otherwise, the proof is finished. Now, by hypothesis $\left(C_{1}\right)$, it follows that Eq. (23) has no solutions for $(x, \lambda) \in$ $\partial \Omega \times(0,1]$. For $\lambda=0$, Eq. (22) is equivalent to the following PBVP:

$$
\left\{\begin{array}{l}
D_{0^{+}}^{\beta}\left(p(t) D_{0^{+}}^{\alpha} x(t)\right)=\frac{\beta}{T^{\beta}} \int_{0}^{T}(T-s)^{\beta-1} f\left(s, x(s), D_{0^{+}}^{\alpha} x(s)\right) d s, \\
x(0)=x(T), \quad D_{0^{+}}^{\alpha} x(0)=D_{0^{+}}^{\alpha} x(T) .
\end{array}\right.
$$


If $x$ is a solution of this PBVP, we have

$$
\frac{\beta}{T^{\beta}} \int_{0}^{T}(T-s)^{\beta-1} f\left(s, x(s), D_{0^{+}}^{\alpha} x(s)\right) d s=0 .
$$

In view of (15), the following equality holds:

$$
x(t)=c \in \operatorname{ker} L, \quad \forall c \in \mathbb{R} .
$$

Thus we have

$$
\left(\left.Q N_{f}\right|_{\operatorname{ker} L}\right) x(t)=\frac{\beta}{T^{\beta}} \int_{0}^{T}(T-s)^{\beta-1} f(s, c, 0) d s=0,
$$

which together with hypothesis $\left(C_{2}\right)$ implies that $x=c \notin \partial \Omega$. So we prove that (23) has no solution for $(x, \lambda) \in \partial \Omega \times[0,1]$. Then, for each $\lambda \in[0,1]$, the Leray-Schauder degree $\operatorname{deg}\left(I-G_{f}(\cdot, \lambda), \Omega, 0\right)$ is well defined. By the homotopy property of degree, we have that

$$
\operatorname{deg}\left(I-G_{f}(\cdot, 1), \Omega, 0\right)=\operatorname{deg}\left(I-G_{f}(\cdot, 0), \Omega, 0\right) .
$$

It is clear that equation $x=G_{f}(x, 1)$ is equivalent to the equation $L x=N_{f} x$. Let us consider the equation $x=G_{f}(x, 1)$, which will have at least one solution if $\operatorname{deg}\left(I-G_{f}(\cdot, 0), \Omega, 0\right) \neq 0$ holds. From now on, we will check this. By the definition of $G_{f}$, we have that

$$
G_{f}(x, 0)=P x+Q N_{f} x+\mathcal{K}(0)=P x+Q N_{f} x .
$$

Obviously, we show that $x=G_{f}(x, 0)=c$ holds for $\forall c \in \mathbb{R}$, which implies that

$$
x-G_{f}(x, 0)=-\frac{\beta}{T^{\beta}} \int_{0}^{T}(T-s)^{\beta-1} f(s, c, 0) d s .
$$

That is,

$$
I-G_{f}(\cdot, 0)=-\left.Q N_{f}\right|_{\text {ker } L}
$$

Then, by applying the Leray-Schauder degree theory, we have

$$
\operatorname{deg}\left(I-G_{f}(\cdot, 0), \Omega, 0\right)=-\operatorname{deg}\left(\left.Q N_{f}\right|_{\operatorname{ker} L}, \Omega \cap \operatorname{ker} L, 0\right),
$$

where the right-hand side degree is the Brouwer degree.

Based on hypothesis $\left(C_{3}\right)$, the equation $L x=N_{f} x$ has at least one solution in $\operatorname{dom} L \cap \bar{\Omega}$. The proof is complete.

Theorem 1 Let $f:[0, T] \times \mathbb{R}^{2} \rightarrow \mathbb{R}$. Assume that

(Ha1) there exist nonnegative functions $a, b, c \in Y$ such that

$$
|f(t, u, v)| \leq a(t)+b(t)|u|+c(t)|v|, \quad \forall t \in[0, T],(u, v) \in \mathbb{R}^{2}
$$


(Ha2) there exists a constant B $>0$ such that either

$$
u f(t, u, v)>0, \quad \forall t \in[0, T], \quad v \in \mathbb{R}, \quad|u|>B
$$

or

$$
u f(t, u, v)<0, \quad \forall t \in[0, T], \quad v \in \mathbb{R}, \quad|u|>B .
$$

Then PBVP (1) has at least one solution, provided that

$$
\gamma=\frac{2 T^{\beta}}{M \Gamma(\beta+1)}\left[\frac{2 T^{\alpha}\|b\|_{\infty}}{\Gamma(\alpha+1)}+\|c\|_{\infty}\right]<1
$$

Proof Let

$$
\Omega_{1}=\left\{x \in \operatorname{dom} L \backslash \operatorname{ker} L \mid L x=\lambda N_{f} x, \lambda \in(0,1)\right\} .
$$

For $x \in \Omega_{1}$, we get $N_{f} x \in \operatorname{Im} L$. It follows from (16) that

$$
\int_{0}^{T}(T-s)^{\beta-1} f\left(s, x(s), D_{0^{+}}^{\alpha} x(s)\right) d s=0 .
$$

By the integral mean value theorem, there exists a constant $\xi \in(0, T)$ such that

$$
f\left(\xi, x(\xi), D_{0^{+}}^{\alpha} x(\xi)\right)=0 .
$$

So, from (Ha2), we get $|x(\xi)| \leq B$. By Lemma 1 , we find that

$$
x(t)=x(\xi)-I_{\xi^{+}}^{\alpha} D_{0^{+}}^{\alpha} x(\xi)+I_{\xi^{+}}^{\alpha} D_{0^{+}}^{\alpha} x(t)
$$

which together with

$$
\begin{aligned}
\left|I_{\xi^{+}}^{\alpha} D_{0^{+}}^{\alpha} x(t)\right| & =\frac{1}{\Gamma(\alpha)}\left|\int_{0}^{t}(t-s)^{\alpha-1} D_{0^{+}}^{\alpha} x(s) d s\right| \\
& \leq \frac{1}{\Gamma(\alpha)}\left\|D_{0^{+}}^{\alpha} x\right\|_{\infty} \cdot \frac{1}{\alpha} t^{\alpha} \\
& \leq \frac{T^{\alpha}}{\Gamma(\alpha+1)}\left\|D_{0^{+}}^{\alpha} x\right\|_{\infty^{\prime}} \quad \forall t \in[0, T]
\end{aligned}
$$

and $|x(\xi)| \leq B$ implies that

$$
\|x\|_{\infty} \leq B+\frac{2 T^{\alpha}}{\Gamma(\alpha+1)}\left\|D_{0^{+}}^{\alpha} x\right\|_{\infty}
$$


Combining hypothesis (Ha1) with (28), $\forall t \in[0, T]$, we get

$$
\begin{aligned}
\mid I_{0^{+}}^{\beta} & N_{f} x(t) \mid \\
& \leq \frac{1}{\Gamma(\beta)} \int_{0}^{t}(t-s)^{\beta-1}\left|f\left(s, x(s), D_{0^{+}}^{\alpha} x(s)\right)\right| d s \\
& \leq \frac{1}{\Gamma(\beta)} \int_{0}^{t}(t-s)^{\beta-1}\left(a(s)+b(s)|x(s)|+c(s)\left|D_{0^{+}}^{\alpha} x(s)\right|\right) d s \\
& \leq \frac{1}{\Gamma(\beta)}\left(\|a\|_{\infty}+\|b\|_{\infty}\|x\|_{\infty}+\|c\|_{\infty}\left\|D_{0^{+}}^{\alpha} x\right\|_{\infty}\right) \cdot \frac{1}{\beta} t^{\beta} \\
& \leq \frac{T^{\beta}\left(\|a\|_{\infty}+\|b\|_{\infty}\left(B+\frac{2 T^{\alpha}}{\Gamma(\alpha+1)}\left\|D_{0^{+}}^{\alpha} x\right\|_{\infty}\right)+\|c\|_{\infty}\left\|D_{0^{+}}^{\alpha} x\right\|_{\infty}\right)}{\Gamma(\beta+1)} .
\end{aligned}
$$

In fact, owing to the fact that $L x=\lambda N_{f} x$, in view of Lemma 1, we have

$$
x(t)=d_{2}+I_{0^{+}}^{\alpha}\left[\frac{d_{1}+\lambda I_{0^{+}}^{\beta} N_{f} x(t)}{p(t)}\right], \quad \forall d_{1}, d_{2} \in \mathbb{R} .
$$

Then, by the boundary condition $x(0)=x(T)$, it follows that

$$
\frac{1}{\Gamma(\alpha)} \int_{0}^{T}(T-s)^{\alpha-1}\left[\frac{d_{1}+\lambda I_{0^{+}}^{\beta} N_{f} x(s)}{p(s)}\right] d s=0 .
$$

Thus, there exists a constant $\eta \in(0, T)$ such that $d_{1}+\lambda I_{0^{+}}^{\beta} N_{f} x(\eta)=0$, which implies

$$
d_{1}=-\lambda I_{0^{+}}^{\beta} N_{f} x(\eta)
$$

As a consequence, we have

$$
p(t) D_{0^{+}}^{\alpha} x(t)=-\lambda I_{0^{+}}^{\beta} N_{f} x(\eta)+\lambda I_{0^{+}}^{\beta} N_{f} x(t) .
$$

Based on (29), it follows that

$$
\begin{aligned}
& \left\|D_{0^{+}}^{\alpha} x\right\|_{\infty} \\
& \quad \leq \frac{2 T^{\beta}}{\Gamma(\beta+1) M}\left[\|a\|_{\infty}+\|b\|_{\infty}\left(B+\frac{2 T^{\alpha}}{\Gamma(\alpha+1)}\left\|D_{0^{+}}^{\alpha} x\right\|_{\infty}\right)+\|c\|_{\infty}\left\|D_{0^{+}}^{\alpha} x\right\|_{\infty}\right] .
\end{aligned}
$$

Thus, from (27), we find that

$$
\left\|D_{0^{+}}^{\alpha} x\right\|_{\infty} \leq \frac{2 T^{\beta}\left(\|a\|_{\infty}+B\|b\|_{\infty}\right)}{(1-\gamma) \Gamma(\beta+1) M}:=M_{2},
$$

which together with (28) yields that

$$
\|x\|_{\infty} \leq B+\frac{2 T^{\alpha} M_{2}}{\Gamma(\alpha+1)}
$$

Therefore, based on (30) and (31), we obtain that

$$
\|x\|_{X}=\max \left\{\|x\|_{\infty},\left\|D_{0^{+}}^{\alpha} x\right\|_{\infty}\right\}=\max \left\{M_{2}, B+\frac{2 T^{\alpha} M_{2}}{\Gamma(\alpha+1)}\right\}:=M_{3} .
$$


It means that $\Omega_{1}$ is bounded. Next, we let $\Omega_{2}=\left\{x \in \operatorname{ker} L \mid Q N_{f} x=0\right\}$. For $x \in \Omega_{2}$, we have $x(t)=d, \forall d \in \mathbb{R}$, which implies that

$$
\int_{0}^{T}(T-s)^{\beta-1} f(s, c, 0) d s=0 .
$$

In view of (Ha2), it follows that $|d| \leq B$. Thus, we obtain

$$
\|x\|_{X} \leq \max \{B, 0\}=B .
$$

That is, $\Omega_{2}$ is bounded. In addition, if (25) holds, set

$$
\Omega_{3}=\left\{x \in \operatorname{ker} L \mid \lambda I x+(1-\lambda) Q N_{f} x=0, \lambda \in[0,1]\right\} .
$$

For $x \in \Omega_{3}$, we have $x(t)=c, \forall c \in \mathbb{R}$ and

$$
\lambda c+(1-\lambda) \frac{\beta}{T^{\beta}} \int_{0}^{T}(T-s)^{\beta-1} f(s, c, 0) d s=0 .
$$

If $\lambda=0$, then $|c| \leq B$ since (25) holds. If $\lambda \in(0,1]$, we can also show that $|c| \leq B$. Otherwise, we get

$$
\lambda c^{2}+(1-\lambda) \frac{\beta}{T^{\beta}} \int_{0}^{T}(T-s)^{\beta-1} c f(s, c, 0) d s>0,
$$

which contradicts (32). So $\Omega_{3}$ is bounded. If (26) holds, let

$$
\Omega_{3}^{\prime}=\left\{x \in \operatorname{ker} L \mid-\lambda I x+(1-\lambda) Q N_{f} x=0, \lambda \in[0,1]\right\} .
$$

By an argument similar to that above, we can prove that $\Omega_{3}^{\prime}$ is also bounded.

Now, it remains to prove that all the conditions of Lemma 7 are satisfied. As for the details, we refer the readers to [29].

As a consequence of Lemma 7, the operator equation $L x=N_{f} x$ has at least one solution in $\operatorname{dom} L \cap \bar{\Omega}$. That is, PBVP (1) has at least one solution in $X_{T}$. The proof is complete.

\section{Existence for PBVP (3)}

\subsection{Preliminaries}

In the following, we provide the necessary background definitions on Fredholm operators and cones in a Banach space (see [28]).

Let $X_{1}, Y_{1}$ be real Banach spaces. Consider a linear mapping $L_{1}: \operatorname{dom} L_{1} \subset X_{1} \rightarrow Y_{1}$ and a nonlinear operator $N_{1}: X_{1} \rightarrow Y_{1}$. Assume that

(A1) $L_{1}$ is a Fredholm operator of index zero; that is, $\operatorname{Im} L_{1}$ is closed and

$$
\operatorname{deg} \operatorname{ker} L_{1}=\operatorname{codim} \operatorname{Im} L_{1}<\infty .
$$

This assumption implies that there exist continuous projections $P_{1}: X_{1} \rightarrow X_{1}$ and $Q_{1}: Y_{1} \rightarrow Y_{1}$ such that $\operatorname{Im} P_{1}=\operatorname{ker} L_{1}$ and $\operatorname{ker} Q_{1}=\operatorname{Im} L_{1}$. Moreover, since $\operatorname{deg} \operatorname{Im} Q_{1}=$ $\operatorname{codim} \operatorname{Im} L_{1}$, there exists an isomorphism $J: \operatorname{Im} Q_{1} \rightarrow \operatorname{ker} L_{1}$. Denote by $L_{P}$ the restriction 
of $L_{1}$ to $\operatorname{ker} P_{1} \cap \operatorname{dom} L_{1}$. Clearly, $L_{P}$ is an isomorphism from $\operatorname{ker} P_{1} \cap \operatorname{dom} L_{1}$ to $\operatorname{Im} L_{1}$, we denote its inverse by $K_{p}: \operatorname{Im} L_{1} \rightarrow \operatorname{ker} P_{1} \cap \operatorname{dom} L_{1}$. It is known that the coincidence equation $L_{1} x=N x$ is equivalent to

$$
x=\left(P_{1}+J Q_{1} N_{1}\right) x+K_{P}\left(I-Q_{1}\right) N_{1} x .
$$

Let $C_{1}$ be a cone in $X_{1}$ such that

(i) $\mu x \in C_{1}$ for all $x \in C_{1}$ and $\mu \geq 0$,

(ii) $x,-x \in C_{1}$ implies $x=\theta$.

It is well known that $C_{1}$ induces a partial order in $X_{1}$ by $x \preceq y$ if and only if $y-x \in C_{1}$. The following property is valid for every cone in a Banach space $X_{1}$.

Lemma 8 Let $C_{1}$ be a cone in $X_{1}$. Then, for every $u \in C_{1}\{0\}$, there exists a positive number $\sigma(u)$ such that

$$
\|x+u\| \geq \sigma(u)\|u\| \quad \text { for all } x \in C_{1} .
$$

Let $\gamma: X_{1} \rightarrow C_{1}$ be a retraction; that is, a continuous mapping such that $\gamma(x)=x$ for all $x \in C_{1}$. Set

$$
\Psi:=P_{1}+J Q_{1} N_{1}+K_{P}\left(I-Q_{1}\right) N_{1} \quad \text { and } \quad \Psi_{\gamma}:=\Psi \circ \gamma .
$$

Theorem 2 ([30]) Let $C_{1}$ be a cone in $X_{1}$, and let $\Omega_{1}, \Omega_{2}$ be open bounded subsets of $X_{1}$ with $\bar{\Omega}_{1} \subset \Omega_{2}$ and $C_{1} \cap\left(\bar{\Omega}_{2} \backslash \Omega_{1}\right) \neq \emptyset$. Assume (A1) and the following assumptions hold:

(A2) $Q_{1} N_{1}: X_{1} \rightarrow Y_{1}$ is continuous and bounded and $K_{P}\left(I-Q_{1}\right) N_{1}: X_{1} \rightarrow X_{1}$ is compact on every bounded subset of $X_{1}$;

(A3) $L_{1} x \neq \lambda N_{1} x$ for all $x \in C_{1} \cap \partial \Omega_{2} \cap \operatorname{Im} L_{1}$ and $\lambda \in(0,1)$;

(A4) $\gamma$ maps subsets of $\bar{\Omega}_{2}$ into bounded subsets of $C_{1}$;

(A5) $\operatorname{deg}\left\{\left.\left[I-\left(P_{1}+J Q_{1} N_{1}\right)_{\gamma}\right]\right|_{\operatorname{ker} L_{1}}, \operatorname{ker} L_{1} \cap \Omega_{2}, 0\right\} \neq 0$;

(A6) there exists $u_{0} \in C_{1}\{0\}$ such that $\|x\| \leq \sigma\left(u_{0}\right)\|\Psi x\|$ for $x \in C_{1}\left(u_{0}\right) \cap \partial \Omega_{1}$, where $C_{1}\left(u_{0}\right)=\left\{x \in C_{1}: \mu u_{0} \preceq x\right.$ for some $\left.\mu>0\right\}$ and $\sigma\left(u_{0}\right)$ such that $\left\|x+u_{0}\right\| \geq \sigma\left(u_{0}\right)\|x\|$ for every $x \in C_{1}$

(A7) $\left(P_{1}+J Q_{1} N_{1}\right) \gamma\left(\partial \Omega_{2}\right) \subset C_{1}$;

(A8) $\Psi_{\gamma}\left(\bar{\Omega}_{2} \backslash \Omega_{1}\right) \subset C_{1}$.

Then the equation $L_{1} x=N_{1} x$ has a solution in the set $C_{1} \cap\left(\bar{\Omega}_{2} \backslash \Omega_{1}\right)$.

\subsection{Main result}

In this subsection, we prove the existence result for PBVP (3). We use the Banach space $Y_{1}=C([0, T], \mathbb{R})$ with the norm $\|y\|_{\infty}=\max _{t \in[0, T]}|y(t)|$ and denote $X_{1}=\left\{x \mid x, D_{0^{+}}^{\alpha} x \in Y_{1}\right\}$ with the norm $\|x\|=\max \left\{\|x\|_{\infty},\left\|D_{0^{+}}^{\alpha} x\right\|_{\infty}\right\}$.

Define the operator $L_{1}: \operatorname{dom} L_{1} \rightarrow X_{1}$ by $L_{1} x=D_{0+}^{\beta} D_{0+}^{\alpha} x$, where

$$
\begin{aligned}
& \operatorname{dom} L_{1} \\
& \quad=\left\{x \in X_{1}: D_{0+}^{\beta} D_{0+}^{\alpha} x \in Y_{1}, x(0)=x(T), x^{\prime}(0)=x^{\prime}(T), D_{0+}^{\alpha} x(0)=D_{0+}^{\alpha} x(T)\right\} .
\end{aligned}
$$


Define the operator $N_{1}: X_{1} \rightarrow Y_{1}$ by $N_{1} x(t)=g\left(t, x(t), D_{0+}^{\alpha} x(t)\right)$. Then problem (3) can be written by $L_{1} x=N_{1} x, x \in \operatorname{dom} L_{1}$. For convenience, we set

$$
G(t, s)=\left\{\begin{aligned}
1+ & \frac{T^{\beta}(t-s)^{\alpha+\beta-1}}{\beta \Gamma(\alpha+\beta)}-\frac{t^{\alpha} T^{\beta-\alpha+1}(T-s)^{\alpha+\beta-2}}{\alpha \beta \Gamma(\alpha+\beta-1)} \\
& -\frac{\Gamma(\beta+1)(T-s)^{\alpha+2 \beta-1}}{\beta \Gamma(\alpha+2 \beta)}+\frac{T^{\beta+1} \Gamma(\beta+1) \Gamma(\alpha)(T-s)^{\alpha+\beta-2}}{\beta \Gamma(\alpha+\beta+1) \Gamma(\alpha+\beta-1)} \\
& +\frac{T^{\beta}(T-s)^{\alpha+\beta-1}}{\beta(\beta+1) \Gamma(\alpha+\beta)}-\frac{T^{\beta+1}(T-s)^{\alpha+\beta-2}}{\alpha \beta(\beta+1) \Gamma(\alpha+\beta-1)} \\
& -\frac{t T^{\beta-1}(T-s)^{\alpha+\beta-1}}{\beta \Gamma(\alpha+\beta)}+\frac{t T^{\beta}(T-s)^{\alpha+\beta-2}}{\alpha \beta \Gamma(\alpha+\beta-1)}+q(t), \\
& 0 \leq s<t \leq T, \\
1- & \frac{t^{\alpha} T^{\beta-\alpha+1}(T-s)^{\alpha+\beta-2}}{\alpha \beta \Gamma(\alpha+\beta-1)}-\frac{\Gamma(\beta+1)(T-s)^{\alpha+2 \beta-1}}{\beta \Gamma(\alpha+2 \beta)} \\
& +\frac{T^{\beta+1} \Gamma(\beta+1) \Gamma(\alpha)(T-s)^{\alpha+\beta-2}}{\beta \Gamma(\alpha+\beta+1) \Gamma(\alpha+\beta-1)}+\frac{T^{\beta}(T-s)^{\alpha+\beta-1}}{\beta(\beta+1) \Gamma(\alpha+\beta)} \\
& -\frac{T^{\beta+1}(T-s)^{\alpha+\beta-2}}{\alpha \beta(\beta+1) \Gamma(\alpha+\beta-1)}-\frac{t T^{\beta-1}(T-s)^{\alpha+\beta-1}}{\beta \Gamma(\alpha+\beta)} \\
& +\frac{t T^{\beta}(T-s)^{\alpha+\beta-2}}{\alpha \beta \Gamma(\alpha+\beta-1)}+q(t), \\
& 0 \leq t<s \leq T,
\end{aligned}\right.
$$

where

$$
\begin{aligned}
q(t)= & \frac{-\alpha t^{\beta}+(\alpha+\beta) T^{\beta}}{\alpha \Gamma(\alpha+\beta+1)} t^{\alpha}+\frac{\Gamma(\beta+1) T^{\alpha}}{\Gamma(\alpha+2 \beta) \Gamma(\alpha+\beta)}-\frac{\beta t T^{\alpha+\beta-1}}{\alpha \Gamma(\alpha+\beta+1)} \\
& -T^{\alpha+\beta}\left(\frac{\Gamma(\alpha+1) \Gamma(\beta+2)-\beta \Gamma(\alpha+\beta)}{\alpha(\beta+1) \Gamma(\alpha+\beta) \Gamma(\alpha+\beta+1)}\right) .
\end{aligned}
$$

Denote a constant $\kappa \in(0,1)$ satisfying

$$
\kappa G(t, s)<1
$$

Lemma 9 The mapping $L_{1}: \operatorname{dom} L_{1} \subset X_{1}$ is a Fredholm operator of index zero. Furthermore, the operator $K_{P}: \operatorname{Im} L_{1} \rightarrow \operatorname{dom} L_{1} \cap \operatorname{ker} P_{1}$ can be written by

$$
K_{P} y(t)=\int_{0}^{1} k(t, s) y(s) d s, \quad t \in[0, T],
$$

where

$$
k(t, s)=\left\{\begin{array}{c}
\frac{(t-s)^{\alpha+\beta-1}}{\Gamma(\alpha+\beta)}-\frac{t^{\alpha}(T-s)^{\alpha+\beta-2}}{\alpha T^{\alpha-1} \Gamma(\alpha+\beta-1)}-\frac{\Gamma(\beta+1)(T-s)^{\alpha+2 \beta-1}}{T^{\beta} \Gamma(\alpha+2 \beta)} \\
\quad+\frac{T \Gamma(\beta+1) \Gamma(\alpha)(T-s)^{\alpha+\beta-2}}{\Gamma(\alpha+\beta+1) \Gamma(\alpha+\beta-1)}+\frac{(T-s)^{\alpha+\beta-1}}{(\beta+1) \Gamma(\alpha+\beta)} \\
\quad-\frac{T(T-s)^{\alpha+\beta-2}}{\alpha(\beta+1) \Gamma(\alpha+\beta-1)}-\frac{t(T-s)^{\alpha+\beta-1}}{T \Gamma(\alpha+\beta)}+\frac{t(T-s)^{\alpha+\beta-2}}{\alpha \Gamma(\alpha+\beta-1)} \\
0 \leq s<t \leq T, \\
-\frac{t^{\alpha}(T-s)^{\alpha+\beta-2}}{\alpha T^{\alpha-1} \Gamma(\alpha+\beta-1)}-\frac{\Gamma(\beta+1)(T-s)^{\alpha+2 \beta-1}}{T^{\beta} \Gamma(\alpha+2 \beta)} \\
\quad+\frac{T \Gamma(\beta+1) \Gamma(\alpha)(T-s)^{\alpha+\beta-2}}{\Gamma(\alpha+\beta+1) \Gamma(\alpha+\beta-1)}+\frac{(T-s)^{\alpha+\beta-1}}{(\beta+1) \Gamma(\alpha+\beta)} \\
-\frac{T(T-s)^{\alpha+\beta-2}}{\alpha(\beta+1) \Gamma(\alpha+\beta-1)}-\frac{t(T-s)^{\alpha+\beta-1}}{T \Gamma(\alpha+\beta)}+\frac{t(T-s)^{\alpha+\beta-2}}{\alpha \Gamma(\alpha+\beta-1)} \\
0 \leq t<s \leq T .
\end{array}\right.
$$

Proof Based on Lemma 1, the solution $x(t)$ of $D_{0+}^{\beta} D_{0_{+}}^{\alpha} x(t)=0$ satisfies $D_{0+}^{\alpha} x(t)=c$. In this case, $c$ should be zero by observing the definition of $D_{0_{+}}^{\alpha}$. Therefore, we have $D_{0_{+}}^{\alpha} x(t)=0$, 
which implies $x(t)=c_{0}+c_{1} t, c_{0}, c_{1} \in \mathbb{R}$. According to the boundary value conditions of (3), we have $\operatorname{ker} L_{1}=\{c, c \in \mathbb{R}\} \cong \mathbb{R}^{1}$.

Let $y(t) \in \operatorname{Im} L_{1}$ and assume that there exists a function $x(t) \in \operatorname{dom} L_{1}$ satisfying $L_{1} x(t)=$ $y(t)$. In view of Lemmas 1 and 2 , we have

$$
x(t)=I_{0+}^{\alpha}\left(I_{0+}^{\beta} y(t)+c_{0}\right)+c_{1}+c_{2} t .
$$

From $D_{0+}^{\alpha} x(0)=D_{0+}^{\alpha} x(T)$, it implies that $\int_{0}^{T}(T-s)^{\beta-1} y(s) d s=0$. On the other hand, suppose $y \in Y_{1}$ satisfying $\int_{0}^{T}(T-s)^{\beta-1} y(s) d s=0$. Let

$$
x(t)=I_{0+}^{\alpha+\beta} y(t)-\left(\frac{1}{T} I_{0+}^{\alpha+\beta} y(T)-\frac{1}{\alpha} I_{0+}^{\alpha+\beta-1} y(T)\right) t-\frac{1}{\alpha T^{\alpha-1}} I_{0+}^{\alpha+\beta-1} y(T) t^{\alpha} .
$$

By a simple calculation, we can prove $x(0)=x(T), x^{\prime}(0)=x^{\prime}(T), D_{0+}^{\alpha} x(0)=D_{0+}^{\alpha} x(T)$, which means $x(t) \in \operatorname{dom} L_{1}$. To conclude, we get

$$
\operatorname{Im} L_{1}=\left\{y \in Y_{1}: \int_{0}^{T}(T-s)^{\beta-1} y(s) d s=0\right\} .
$$

Consider the linear operator $P_{1}: X_{1} \rightarrow X_{1}$ defined by

$$
P_{1} x(t)=\frac{\beta}{T^{\beta}} \int_{0}^{T}(T-s)^{\beta-1} x(s) d s, \quad t \in[0, T],
$$

and the operator $Q_{1}: Y_{1} \rightarrow Y_{1}$ defined by

$$
Q_{1} y(t)=\frac{\beta}{T^{\beta}} \int_{0}^{T}(T-s)^{\beta-1} y(s) d s, \quad t \in[0, T] .
$$

For $x(t) \in X_{1}$, we get

$$
P_{1}\left(P_{1} x\right)=P_{1}\left[\frac{\beta}{T^{\beta}} \int_{0}^{T}(T-s)^{\beta-1} x(s) d s\right]=\frac{\beta}{T^{\beta}} \int_{0}^{T}(T-s)^{\beta-1} x(s) d s=P_{1} x .
$$

Hence, we have $P_{1}^{2}=P_{1}$. Similarly, we can get $Q_{1}^{2}=Q_{1}$. Note that $\operatorname{Im} P_{1}=\operatorname{ker} L_{1}$ and $\operatorname{ker} Q_{1}=$ $\operatorname{Im} L_{1}$. It follows from

$$
\operatorname{Ind} L_{1}=\operatorname{deg} \operatorname{ker} L_{1}-\operatorname{codim} \operatorname{Im} L_{1}=0
$$

that $L_{1}$ is a Fredholm mapping of index zero.

It remains to prove that the operator $K_{P}$ is the inverse of $\left.L_{1}\right|_{\operatorname{dom} L_{1} \cap \operatorname{ker} P_{1}}$.

In fact, for $x(t) \in \operatorname{dom} L_{1} \cap \operatorname{ker} P_{1}$, we have $D_{0+}^{\beta} D_{0_{+}}^{\alpha} x(t)=y(t)$. By Lemma 1 , we have $x(t)=$ $I_{0+}^{\alpha}\left(I_{0+}^{\beta} y(t)+c_{0}\right)+c_{1}+c_{2} t$. According to $x(0)=x(T), x^{\prime}(0)=x^{\prime}(T), D_{0+}^{\alpha} x(0)=D_{0+}^{\alpha} x(T)$, we get

$$
c_{0}=-\frac{\Gamma(\alpha)}{T^{\alpha-1}} I_{0+}^{\alpha+\beta-1} y(T), \quad c_{2}=-\frac{1}{T} I_{0+}^{\alpha+\beta} y(T)+\frac{1}{\alpha} I_{0+}^{\alpha+\beta-1} y(T) .
$$

Since $x(t) \in \operatorname{ker} P_{1}$, i.e., $\frac{\beta}{T^{\beta}} \int_{0}^{T}(T-s)^{\beta-1} x(s) d s=0$, we obtain

$$
c_{1}=-\frac{\Gamma(\beta+1)}{T^{\beta}} I_{0+}^{2 \beta+\alpha} y(T)-\frac{T^{\alpha} \Gamma(\beta+1)}{\Gamma(\alpha+\beta+1)} c_{0}-\frac{T}{\beta+1} c_{2} .
$$


Define an operator

$$
K_{P} y(t)=I_{0+}^{\alpha}\left(I_{0+}^{\beta} y(t)+c_{0}\right)+c_{1}+c_{2} t
$$

Substituting $c_{0}, c_{1}, c_{2}$ in the above equality, we obtain

$$
\begin{aligned}
& K_{P} y(t)=I_{0+}^{\alpha}\left(I_{0+}^{\beta} y(t)+c_{0}\right)+c_{1}+c_{2} t \\
& =I_{0+}^{\alpha+\beta} y(t)-\frac{1}{\alpha T^{\alpha-1}} I_{0+}^{\alpha+\beta-1} y(T) t^{\alpha}-\frac{\Gamma(\beta+1)}{T^{\beta}} I_{0+}^{\alpha+2 \beta} y(T) \\
& +\frac{T \Gamma(\beta+1) \Gamma(\alpha)}{\Gamma(\alpha+\beta+1)} I_{0+}^{\alpha+\beta-1} y(T)+\frac{1}{\beta+1} I_{0+}^{\alpha+\beta} y(T) \\
& -\frac{T}{\alpha(\beta+1)} I_{0+}^{\alpha+\beta-1} y(T)-\left(\frac{1}{T} I_{0+}^{\alpha+\beta} y(T)-\frac{1}{\alpha} I_{0+}^{\alpha+\beta-1} y(T)\right) t \\
& =\frac{1}{\Gamma(\alpha+\beta)} \int_{0}^{t}(t-s)^{\alpha+\beta-1} y(s) d s \\
& -\frac{t^{\alpha}}{\alpha T^{\alpha-1} \Gamma(\alpha+\beta-1)} \int_{0}^{T}(T-s)^{\alpha+\beta-2} y(s) d s \\
& -\frac{\Gamma(\beta+1)}{T^{\beta} \Gamma(\alpha+2 \beta)} \int_{0}^{T}(T-s)^{\alpha+2 \beta-1} y(s) d s \\
& +\frac{T \Gamma(\beta+1) \Gamma(\alpha)}{\Gamma(\alpha+\beta+1) \Gamma(\alpha+\beta-1)} \int_{0}^{T}(T-s)^{\alpha+\beta-2} y(s) d s \\
& +\frac{1}{(\beta+1) \Gamma(\alpha+\beta)} \int_{0}^{T}(T-s)^{\alpha+\beta-1} y(s) d s \\
& -\frac{T}{\alpha(\beta+1) \Gamma(\alpha+\beta-1)} \int_{0}^{T}(T-s)^{\alpha+\beta-2} y(s) d s \\
& -\frac{t}{T \Gamma(\alpha+\beta)} \int_{0}^{T}(T-s)^{\alpha+\beta-1} y(s) d s \\
& +\frac{t}{\alpha \Gamma(\alpha+\beta-1)} \int_{0}^{T}(T-s)^{\alpha+\beta-2} y(s) d s \\
& =\int_{0}^{T} k(t, s) y(s) d s .
\end{aligned}
$$

It can be shown that $L_{1} K_{P} y(t)=y(t)$, which implies $K_{P}=\left(\left.L_{1}\right|_{\operatorname{dom} L_{1} \cap \operatorname{ker} P_{1}}\right)^{-1}$. This completes the proof of Lemma 9.

Lemma 10 Assume that $\Omega \subset X_{1}$ is an open bounded set such that $\operatorname{dom}\left(L_{1}\right) \cap \bar{\Omega} \neq \emptyset$, then $N_{1}$ is L-compact on $\bar{\Omega}$.

Proof Based on the continuity of $g$, we obtain that $Q_{1} N_{1}(\bar{\Omega})$ and $K_{P}\left(I-Q_{1}\right) N_{1}(\bar{\Omega})$ are bounded. Hence, for $x(t) \in \bar{\Omega}, t \in[0, T]$, there exists a positive constant $M$ such that $\left|\left(I-Q_{1}\right) N_{1} x(t)\right| \leq M,\left|\frac{1}{\alpha T^{\alpha-1}} I_{0+}^{\alpha+\beta-1}\left(I-Q_{1}\right) N_{1} x(T)\right| \leq M$ and $\mid \frac{1}{T} I_{0+}^{\alpha+\beta}\left(I-Q_{1}\right) N_{1} x(T)-$ $\frac{1}{\alpha} I_{0+}^{\alpha+\beta-1}\left(I-Q_{1}\right) N_{1} x(T) \mid \leq M$. In view of the Arzela-Ascoli theorem, we need only to prove that $K_{P}\left(I-Q_{1}\right) N_{1}(\bar{\Omega})$ is equicontinuous. 
For $0 \leq t_{1}<t_{2} \leq T, x \in \bar{\Omega}$, by virtue of the definition of $K_{P}$, we have

$$
\begin{aligned}
&\left|K_{P}\left(I-Q_{1}\right) N_{1} x\left(t_{2}\right)-K_{P}\left(I-Q_{1}\right) N_{1} x\left(t_{1}\right)\right| \\
&=\mid\left[I_{0+}^{\alpha+\beta}\left(I-Q_{1}\right) N_{1} x(t)\right]_{t=t_{2}}+\frac{c_{0}}{\Gamma(\alpha+1)} t_{2}^{2}+c_{1}+c_{2} t_{2} \\
& \quad-\left[I_{0+}^{\alpha+\beta}\left(I-Q_{1}\right) N_{1} x(t)\right]_{t=t_{1}}-\frac{c_{0}}{\Gamma(\alpha+1)} t_{1}^{2}-c_{1}-c_{2} t_{1} \mid \\
& \leq \frac{1}{\Gamma(\alpha+\beta)} \mid \int_{0}^{t_{2}}\left(t_{2}-s\right)^{\alpha+\beta-1}\left(I-Q_{1}\right) N_{1} x(s) d s \\
&-\int_{0}^{t_{1}}\left(t_{1}-s\right)^{\alpha+\beta-1}\left(I-Q_{1}\right) N_{1} x(s) d s \mid \\
&+\left|\frac{1}{\alpha T^{\alpha-1}} I_{0+}^{\alpha+\beta-1}\left(I-Q_{1}\right) N_{1} x(T)\right| \cdot\left|t_{2}^{\alpha}-t_{1}^{\alpha}\right| \\
&+\left|\left(\frac{1}{T} I_{0+}^{\alpha+\beta}-\frac{1}{\alpha} I_{0+}^{\alpha+\beta-1}\right)\left(I-Q_{1}\right) N_{1} x(T)\right| \cdot\left|t_{2}-t_{1}\right| \\
& \leq \frac{1}{\Gamma(\alpha+\beta)}\left|\int_{0}^{t_{1}}\left[\left(t_{2}-s\right)^{\alpha+\beta-1}-\left(t_{1}-s\right)^{\alpha+\beta-1}\right]\left(I-Q_{1}\right) N_{1} x(s) d s\right| \\
& \leq \frac{1}{\Gamma(\alpha+\beta+1)}\left[t_{2}^{\alpha+\beta}-t_{1}^{\alpha+\beta}+\left(t_{2}-t_{1}\right)^{\alpha+\beta}\right]+M\left(t_{2}^{\alpha}-t_{1}^{\alpha}+t_{2}-t_{1}\right) . \\
&+\frac{1(\alpha+\beta)}{\Gamma\left(t_{2}\right.}\left(t_{2}-s\right)^{\alpha+\beta-1}\left(I-Q_{1}\right) N_{1} x(s) d s \mid+M\left(t_{2}^{\alpha}-t_{1}^{\alpha}+t_{2}-t_{1}\right) \\
&\left.\mid \frac{1}{\Gamma}\right)
\end{aligned}
$$

Notice that $t$ and $t^{\alpha}$ are uniformly continuous on $[0, T]$. Therefore, we have $K_{P}(I-$ $\left.Q_{1}\right) N_{1}(\bar{\Omega})$ is equicontinuous on $[0, T]$. The proof is completed.

\section{Theorem 3 Assume that}

(Hb1) for $t \in[0, T]$ and $(u, v) \in[0, B] \times[0, B]$, one has

$$
-\kappa(u+v) \leq g(t, u, v) \leq-c_{1} u-c_{2} v+c_{3}
$$

and

$$
g(t, u, v) \leq-b_{1}|g(t, u, v)|+b_{2} u+b_{3} v+b_{4}
$$

where $b_{1}, b_{2}, b_{3}, b_{4}, c_{1}, c_{2}, c_{3}, B$ are positive constants with

$$
\begin{aligned}
& b_{1} c_{1} c_{2} \beta+b_{1} c_{1}^{2} \beta+8 T^{\alpha+\beta-1} b_{2} c_{2}^{2}-8 T^{\alpha+\beta-1} b_{3} c_{1} c_{2}>0, \\
& \Gamma(3-\alpha) \Gamma(\alpha+\beta)-2 \kappa(\alpha-1) T^{\alpha+2 \beta-2}>0 \\
& B>\max \left\{A_{1}, A_{2}, \frac{c_{3}}{c_{1}}\right\}
\end{aligned}
$$

where

$$
A_{1}=\frac{b_{1} c_{2} c_{3} \beta+8 b_{2} c_{2} c_{3} T^{\alpha+\beta-1}+8 b_{4} c_{1} c_{2} T^{\alpha+\beta-1}}{b_{1} c_{1} c_{2} \beta+b_{1} c_{1}^{2} \beta+8 T^{\alpha+\beta-1} b_{2} c_{2}^{2}-8 T^{\alpha+\beta-1} b_{3} c_{1} c_{2}}
$$


and

$$
A_{2}=\frac{c_{3}(2 \alpha+\beta-2) T^{\beta}}{\Gamma(3-\alpha) \Gamma(\alpha+\beta)-2 \kappa(\alpha-1) T^{\alpha+2 \beta-2}} .
$$

(Hb2) there exist $r \in(0, B), t_{0} \in[0, T], m \in(0,1)$, and $h_{i}(x):(0, r] \rightarrow[0,+\infty), i=1,2$, such that $g(t, u, v) \geq h_{1}(u)+h_{2}(v)$ for $t \in[0, T],(u, v) \in(0, r] \times(0, r]$. Moreover, $\frac{h_{1}(u)}{u}$ and $\frac{h_{2}(v)}{v}$ are nonincreasing on $(0, r]$ and

$$
\frac{\beta}{T^{\beta}} \frac{h_{i}(r)}{r} \int_{0}^{T} G\left(t_{0}, s\right)(T-s)^{\beta-1} d s \geq \frac{1-m}{2 m}, \quad i=1,2
$$

Then problem (3) has at least one positive solution on $[0, T]$.

Proof Firstly, conditions (A1) and (A2) of Theorem 3 are satisfied based on Lemmas 9 and 10.

Then, consider the cone $C_{1}=\left\{u \in X_{1}: u(t) \geq 0, D_{0+}^{\alpha} u(t) \geq 0, t \in[0, T]\right\}$. Let $\Omega_{1}=\{u \in$ $\left.X_{1}: m\|u\|<|u(t)|<r, m\|u\|<\left|D_{0+}^{\alpha} u(t)\right|<r, t \in[0, T]\right\}, \Omega_{2}=\left\{u \in X_{1}:\|u\|<B, t \in[0, T]\right\}$. Obviously, $\Omega_{1}$ and $\Omega_{2}$ are bounded and

$$
\bar{\Omega}_{1}=\left\{u \in X_{1}: m\|u\| \leq|u(t)| \leq r, m\|u\| \leq\left|D_{0+}^{\alpha} u(t)\right| \leq r, t \in[0, T]\right\} \subset \Omega_{2} .
$$

Furthermore, $C_{1} \cap\left(\bar{\Omega}_{2} \backslash \Omega_{1}\right) \neq \emptyset$. Let $J=I$ and $(\gamma u)(t)=|u(t)|$ for $u \in X_{1}$, then $\gamma$ is a retraction and maps subsets of $\bar{\Omega}_{2}$ into bounded subsets of $C_{1}$, which means that (A4) holds.

Next, we will prove that (A3) holds. Suppose that there exist $x_{0} \in \partial \Omega_{2} \cap C_{1} \cap \operatorname{dom} L_{1}$ and $\lambda_{0} \in(0,1)$ such that $L_{1} x_{0}=\lambda_{0} N_{1} x_{0}$, that is, $D_{0+}^{\beta} D_{0+}^{\alpha} x_{0}(t)=\lambda_{0} g\left(t, x_{0}(t), D_{0+}^{\alpha} x_{0}(t)\right), t \in[0, T]$. Then assumption ( $\mathrm{Hb} 1)$ gives

$$
\begin{aligned}
D_{0+}^{\beta} & D_{0+}^{\alpha} x_{0}(t) \\
& =\lambda_{0} g\left(t, x_{0}(t), D_{0+}^{\alpha} x_{0}(t)\right) \\
& \leq-\lambda_{0} b_{1}\left|g\left(t, x_{0}(t), D_{0+}^{\alpha} x_{0}(t)\right)\right|+\lambda_{0} b_{2} x_{0}(t)+\lambda_{0} b_{3} D_{0+}^{\alpha} x_{0}(t)+\lambda_{0} b_{4} \\
& =-b_{1}\left|\lambda_{0} g\left(t, x_{0}(t), D_{0+}^{\alpha} x_{0}(t)\right)\right|+\lambda_{0} b_{2} x_{0}(t)+\lambda_{0} b_{3} D_{0+}^{\alpha} x_{0}(t)+\lambda_{0} b_{4} \\
& =-b_{1}\left|D_{0+}^{\beta} D_{0+}^{\alpha} x_{0}(t)\right|+\lambda_{0} b_{2} x_{0}(t)+\lambda_{0} b_{3} D_{0+}^{\alpha} x_{0}(t)+\lambda_{0} b_{4} \\
& \leq-b_{1}\left|D_{0+}^{\beta} D_{0+}^{\alpha} x_{0}(t)\right|+b_{2} x_{0}(t)+b_{3} D_{0+}^{\alpha} x_{0}(t)+b_{4}
\end{aligned}
$$

and

$$
D_{0+}^{\beta} D_{0+}^{\alpha} x_{0}(t)=\lambda_{0} g\left(t, x_{0}(t), D_{0+}^{\alpha} x_{0}(t)\right) \leq-\lambda_{0} c_{1} x_{0}(t)-\lambda_{0} c_{2} D_{0+}^{\alpha} x_{0}(t)+\lambda_{0} c_{3} .
$$

Since $D_{0+}^{\beta} D_{0+}^{\alpha} x_{0}(t)=\lambda_{0} g\left(t, x_{0}(t), D_{0+}^{\alpha} x_{0}(t)\right) \in \operatorname{Im} L_{1}$, based on the definition of $\operatorname{Im} L_{1}$ and (38), we can obtain

$$
\begin{aligned}
0 & =\int_{0}^{T}(T-s)^{\beta-1} D_{0+}^{\beta} D_{0+}^{\alpha} x_{0}(s) d s \\
& \leq \int_{0}^{T}(T-s)^{\beta-1}\left(-\lambda_{0} c_{1} x_{0}(s)-\lambda_{0} c_{2} D_{0+}^{\alpha} x_{0}(s)+\lambda_{0} c_{3}\right) d s,
\end{aligned}
$$


which gives

$$
\int_{0}^{T}(T-s)^{\beta-1} x_{0}(s) d s \leq-\frac{c_{2}}{c_{1}} \int_{0}^{T}(T-s)^{\beta-1} D_{0+}^{\alpha} x_{0}(s) d s+\frac{c_{3} T^{\beta}}{c_{1} \beta} .
$$

Furthermore, (37) and (40) imply

$$
\begin{aligned}
0= & \int_{0}^{T}(T-s)^{\beta-1} D_{0_{+}}^{\beta} D_{0+}^{\alpha} x_{0}(s) d s \\
\leq & \int_{0}^{T}(T-s)^{\beta-1}\left[-b_{1}\left|D_{0+}^{\beta} D_{0+}^{\alpha} x_{0}(s)\right|+b_{2} x_{0}(s)+b_{3} D_{0+}^{\alpha} x_{0}(s)+b_{4}\right] d s \\
= & -b_{1} \int_{0}^{T}(T-s)^{\beta-1}\left|D_{0+}^{\beta} D_{0+}^{\alpha} x_{0}(s)\right| d s+b_{2} \int_{0}^{T}(T-s)^{\beta-1} x_{0}(s) d s \\
& +b_{3} \int_{0}^{T}(T-s)^{\beta-1} D_{0+}^{\alpha} x_{0}(s) d s+\frac{b_{4} T^{\beta}}{\beta}
\end{aligned}
$$

which gives

$$
\begin{aligned}
& \int_{0}^{T}(T-s)^{\beta-1}\left|D_{0+}^{\beta} D_{0+}^{\alpha} x_{0}(s)\right| d s \\
& \quad \leq \frac{b_{2}}{b_{1}} \int_{0}^{T}(T-s)^{\beta-1} x_{0}(s) d s+\frac{b_{3}}{b_{1}} \int_{0}^{T}(T-s)^{\beta-1} D_{0+}^{\alpha} x_{0}(s) d s+\frac{b_{4} T^{\beta}}{b_{1} \beta} \\
& \quad \leq\left(-\frac{b_{2} c_{2}}{b_{1} c_{1}}+\frac{b_{3}}{b_{1}}\right) \int_{0}^{T}(T-s)^{\beta-1} D_{0+}^{\alpha} x_{0}(s) d s+\frac{b_{2} c_{3} T^{\beta}}{b_{1} c_{1} \beta}+\frac{b_{4} T^{\beta}}{b_{1} \beta}
\end{aligned}
$$

Based on the function expression of $k(t, s)$, we get

$$
|k(t, s)| \leq 8 T(T-s)^{\alpha+\beta-2}, s, t \in[0, T] .
$$

By virtue of (40), (41), (42), and the equation $x_{0}=\left(I-P_{1}\right) x_{0}+P_{1} x_{0}=K_{P} L_{1}\left(I-P_{1}\right) x_{0}+$ $P_{1} x_{0}=P_{1} x_{0}+K_{P} L_{1} x_{0}$, we have

$$
\begin{aligned}
x_{0}= & P_{1} x_{0}+K_{P} L_{1} x_{0} \\
= & \frac{\beta}{T^{\beta}} \int_{0}^{T}(T-s)^{\beta-1} x_{0}(s) d s+\int_{0}^{T} k(t, s) D_{0+}^{\beta} D_{0+}^{\alpha} x_{0}(s) d s \\
\leq & -\frac{\beta c_{2}}{T^{\beta} c_{1}} \int_{0}^{T}(T-s)^{\beta-1} D_{0+}^{\alpha} x_{0}(s) d s+\frac{c_{3}}{c_{1}} \\
& +\int_{0}^{T}|k(t, s)| \cdot\left|D_{0+}^{\beta} D_{0+}^{\alpha} x_{0}(s)\right| d s \\
\leq & -\frac{\beta c_{2}}{T^{\beta} c_{1}} \int_{0}^{T}(T-s)^{\beta-1} D_{0+}^{\alpha} x_{0}(s) d s+\frac{c_{3}}{c_{1}} \\
& +8 T \int_{0}^{T}(T-s)^{\alpha+\beta-2}\left|D_{0+}^{\beta} D_{0+}^{\alpha} x_{0}(s)\right| d s
\end{aligned}
$$




$$
\begin{aligned}
\leq & -\frac{\beta c_{1}}{T^{\beta} c_{2}} \int_{0}^{T}(T-s)^{\beta-1} D_{0+}^{\alpha} x_{0}(s) d s+\frac{c_{3}}{c_{1}} \\
& +8 T^{\alpha-1} \int_{0}^{T}(T-s)^{\beta-1}\left|D_{0+}^{\beta} D_{0+}^{\alpha} x_{0}(s)\right| d s \\
\leq & \left(-\frac{\beta c_{1}}{T^{\beta} c_{2}}-\frac{8 T^{\alpha-1} b_{2} c_{2}}{b_{1} c_{1}}+\frac{8 T^{\alpha-1} b_{3}}{b_{1}}\right) \int_{0}^{T}(T-s)^{\beta-1} D_{0+}^{\alpha} x_{0}(s) d s+\frac{c_{3}}{c_{1}} \\
& +\frac{8 b_{2} c_{3} T^{\alpha+\beta-1}}{b_{1} c_{1} \beta}+\frac{8 b_{4} T^{\alpha+\beta-1}}{b_{1} \beta} \\
\leq & \left(-\frac{c_{1}}{c_{2}}-\frac{8 T^{\alpha+\beta-1} b_{2} c_{2}}{b_{1} c_{1} \beta}+\frac{8 T^{\alpha+\beta-1} b_{3}}{b_{1} \beta}\right) B+\frac{c_{3}}{c_{1}} \\
& +\frac{8 b_{2} c_{3} T^{\alpha+\beta-1}}{b_{1} c_{1} \beta}+\frac{8 b_{4} T^{\alpha+\beta-1}}{b_{1} \beta} .
\end{aligned}
$$

In view of (Hb1), we have

$$
D_{0+}^{\beta} D_{0+}^{\alpha} x_{0}(t)=\lambda_{0} g\left(t, x_{0}(t), D_{0+}^{\alpha} x_{0}(t)\right) \geq-\lambda_{0} \kappa\left(x_{0}(t)+D_{0+}^{\alpha} x_{0}(t)\right)
$$

and

$$
\begin{aligned}
& D_{0+}^{\beta} D_{0+}^{\alpha} x_{0}(t) \\
& \quad=\lambda_{0} g\left(t, x_{0}(t), D_{0+}^{\alpha} x_{0}(t)\right) \leq-\lambda_{0} c_{1} x_{0}(t)-\lambda_{0} c_{2} D_{0+}^{\alpha} x_{0}(t)+\lambda_{0} c_{3} \leq c_{3} .
\end{aligned}
$$

In addition, based on the definition of function $k(t, s)$, we obtain

$$
\frac{d^{2} k(s, \tau)}{d s^{2}}= \begin{cases}\frac{(s-\tau)^{\alpha+\beta-3}}{\Gamma(\alpha+\beta-2)}-\frac{(\alpha-1) s^{\alpha-2}(T-\tau)^{\alpha+\beta-2}}{T^{\alpha-1} \Gamma(\alpha+\beta-1)}, & 0 \leq \tau<s \leq T, \\ -\frac{(\alpha-1) s^{\alpha-2}(T-\tau)^{\alpha+\beta-2}}{T^{\alpha-1} \Gamma(\alpha+\beta-1)}, & 0 \leq s<\tau \leq T .\end{cases}
$$

Hence, on the basis of (43)-(45), we have

$$
\begin{aligned}
\int_{0}^{T} & \frac{d^{2} k(s, \tau)}{d s^{2}} D_{0+}^{\beta} D_{0+}^{\alpha} x_{0}(\tau) d \tau \\
= & \int_{0}^{s}\left(\frac{(s-\tau)^{\alpha+\beta-3}}{\Gamma(\alpha+\beta-2)}-\frac{(\alpha-1) s^{\alpha-2}(T-\tau)^{\alpha+\beta-2}}{T^{\alpha-1} \Gamma(\alpha+\beta-1)}\right) D_{0+}^{\beta} D_{0+}^{\alpha} x_{0}(\tau) d \tau \\
& +\int_{s}^{T}-\frac{(\alpha-1) s^{\alpha-2}(T-\tau)^{\alpha+\beta-2}}{T^{\alpha-1} \Gamma(\alpha+\beta-1)} D_{0+}^{\beta} D_{0+}^{\alpha} x_{0}(\tau) d \tau \\
\leq & c_{3} \int_{0}^{s}\left|\frac{(s-\tau)^{\alpha+\beta-3}}{\Gamma(\alpha+\beta-2)}-\frac{(\alpha-1) s^{\alpha-2}(T-\tau)^{\alpha+\beta-2}}{T^{\alpha-1} \Gamma(\alpha+\beta-1)}\right| d \tau \\
& +\int_{s}^{T} \frac{\kappa(\alpha-1) s^{\alpha-2}(T-\tau)^{\alpha+\beta-2}}{T^{\alpha-1} \Gamma(\alpha+\beta-1)}\left(x_{0}(\tau)+D_{0+}^{\alpha} x_{0}(\tau)\right) d \tau \\
\leq & c_{3}\left[\frac{s^{\alpha+\beta-2}}{\Gamma(\alpha+\beta-1)}+\frac{s^{\alpha-2}(\alpha-1)\left(T^{\alpha+\beta-1}-(T-s)^{\alpha+\beta-1}\right)}{T^{\alpha-1} \Gamma(\alpha+\beta)}\right] \\
& +\frac{2 \kappa B s^{\alpha-2}(\alpha-1)(T-s)^{\alpha+\beta-1}}{T^{\alpha-1} \Gamma(\alpha+\beta)} \\
\leq & \frac{T^{\alpha+\beta-2}(2 \alpha+\beta-2) c_{3}}{\Gamma(\alpha+\beta)}+\frac{2 \kappa B(\alpha-1) T^{\alpha+\beta-2}}{\Gamma(\alpha+\beta)} .
\end{aligned}
$$


Therefore, by a simple calculation, we get

$$
\begin{aligned}
& D_{0+}^{\alpha} x_{0} \\
& \quad=D_{0+}^{\alpha}\left(P_{1} x_{0}+K_{P} L_{1} x_{0}\right) \\
& \quad=\frac{1}{\Gamma(2-\alpha)} \int_{0}^{t}(t-s)^{1-\alpha} \frac{d^{2}\left(K_{P} L_{1} x_{0}\right)(s)}{d s^{2}} d s \\
& \quad=\frac{1}{\Gamma(2-\alpha)} \int_{0}^{t}(t-s)^{1-\alpha} \int_{0}^{T} \frac{d^{2} k(s, \tau)}{d s^{2}} D_{0+}^{\beta} D_{0+}^{\alpha} x_{0}(\tau) d \tau d s \\
& \quad \leq \frac{1}{\Gamma(2-\alpha)} \int_{0}^{t}(t-s)^{1-\alpha} d s \cdot\left(\frac{T^{\alpha+\beta-2}(2 \alpha+\beta-2) c_{3}}{\Gamma(\alpha+\beta)}+\frac{2 \kappa B(\alpha-1) T^{\alpha+\beta-2}}{\Gamma(\alpha+\beta)}\right) \\
& \quad=\frac{1}{\Gamma(3-\alpha)} T^{2-\alpha} \cdot\left(\frac{T^{\alpha+\beta-2}(2 \alpha+\beta-2) c_{3}}{\Gamma(\alpha+\beta)}+\frac{2 \kappa B(\alpha-1) T^{\alpha+\beta-2}}{\Gamma(\alpha+\beta)}\right) \\
& \quad=\frac{1}{\Gamma(3-\alpha) \Gamma(\alpha+\beta)} T^{\beta} \cdot\left[(2 \alpha+\beta-2) c_{3}+2 \kappa B(\alpha-1) T^{\alpha+\beta-2}\right] .
\end{aligned}
$$

Based on the definition of norm $\|\cdot\|$, we have $B \leq \max \left\{A_{1}, A_{2}\right\}$ with

$$
A_{1}=\frac{b_{1} c_{2} c_{3} \beta+8 b_{2} c_{2} c_{3} T^{\alpha+\beta-1}+8 b_{4} c_{1} c_{2} T^{\alpha+\beta-1}}{b_{1} c_{1} c_{2} \beta+b_{1} c_{1}^{2} \beta+8 T^{\alpha+\beta-1} b_{2} c_{2}^{2}-8 T^{\alpha+\beta-1} b_{3} c_{1} c_{2}}
$$

and

$$
A_{2}=\frac{c_{3}(2 \alpha+\beta-2) T^{\beta}}{\Gamma(3-\alpha) \Gamma(\alpha+\beta)-2 \kappa(\alpha-1) T^{\alpha+2 \beta-2}},
$$

which contradicts (Hb1). Hence (A3) holds.

In order to prove (A5), we consider $x(t) \in \operatorname{ker} L_{1} \cap \bar{\Omega}_{2}$, then $x(t) \equiv c$. For $c \in[-B, B]$ and $\lambda \in[0,1]$, we obtain

$$
\begin{aligned}
& H(c, \lambda) \\
& \quad=\left[I-\lambda\left(P_{1}+J Q_{1} N_{1}\right)_{\gamma}\right] c \\
& \quad=c-\lambda \frac{\beta}{T^{\beta}} \int_{0}^{T}(T-s)^{\beta-1}|c| d s-\lambda \frac{\beta}{T^{\beta}} \int_{0}^{T}(T-s)^{\beta-1} g\left(s,|c|, D_{0+}^{\alpha}|c|\right) d s \\
& \quad=c-\lambda|c|-\lambda \frac{\beta}{T^{\beta}} \int_{0}^{T}(T-s)^{\beta-1} g\left(s,|c|, D_{0+}^{\alpha}|c|\right) d s \\
& \quad=c-\lambda \frac{\beta}{T^{\beta}} \int_{0}^{T}(T-s)^{\beta-1}\left[g\left(s,|c|, D_{0+}^{\alpha}|c|\right)+|c|\right] d s .
\end{aligned}
$$

By use of the proof by contradiction, it can be shown that $H(c, \lambda)=0$ implies $c \geq 0$. Suppose $H(B, \lambda)=0$ for some $\lambda \in(0,1]$, then we have

$$
0=B-\lambda B-\lambda \frac{\beta}{T^{\beta}} \int_{0}^{T}(T-s)^{\beta-1} g\left(s, B, D_{0+}^{\alpha} B\right) d s .
$$

In view of (Hb1), we have

$$
0 \leq B(1-\lambda)=\lambda \frac{\beta}{T^{\beta}} \int_{0}^{T}(T-s)^{\beta-1} g\left(s, B, D_{0+}^{\alpha} B\right) d s \leq \lambda\left(-c_{1} B+c_{3}\right)<0,
$$


which is a contradiction. In addition, if $\lambda=0$, then $B=0$, which is impossible. As a result, for $x \in \operatorname{ker} L_{1} \cap \partial \Omega_{2}$ and $\lambda \in[0,1]$, we have $H(x, \lambda) \neq 0$. Thus,

$$
\begin{aligned}
\operatorname{deg} & \left\{\left[I-\left(P_{1}+J Q_{1} N_{1}\right)_{\gamma}\right]_{\operatorname{ker} L_{1}}, \operatorname{ker} L_{1} \cap \Omega_{2}, 0\right\} \\
& =\operatorname{deg}\left\{H(\cdot, 1), \operatorname{ker} L_{1} \cap \Omega_{2}, 0\right\} \\
& =\operatorname{deg}\left\{H(\cdot, 0), \operatorname{ker} L_{1} \cap \Omega_{2}, 0\right\} \\
& =\operatorname{deg}\left\{I, \operatorname{ker} L_{1} \cap \Omega_{2}, 0\right\} \\
& =1 \neq 0 .
\end{aligned}
$$

So (A5) holds. It remains to prove (A6). Let $x_{0}(t) \equiv 1, t \in[0, T]$, then $x_{0} \in C_{1} \backslash\{0\}, C_{1}\left(x_{0}\right)=$ $\left\{x \in C_{1}: x(t)>0, t \in[0, T]\right\}$. We take $\sigma\left(x_{0}\right)=1$ and let $x \in C_{1}\left(x_{0}\right) \cap \partial \Omega_{1}$, then $0<\|x\| \leq r$ and $x(t) \geq m\|x\|$ on $[0, T]$.

For $u \in C_{1}\left(x_{0}\right) \cap \Omega_{1}$, (Hb2) implies

$$
\begin{aligned}
(\Psi) x & \left(t_{0}\right) \\
= & {\left[\left(P_{1}+J Q_{1} N_{1}+K_{P}\left(I-Q_{1}\right) N_{1}\right) x(t)\right]_{t=t_{0}} } \\
= & {\left[P_{1} x(t)\right]_{t=t_{0}}+\left[\left(J Q_{1} N_{1}+K_{P}\left(I-Q_{1}\right) N_{1}\right) x(t)\right]_{t=t_{0}} } \\
= & \frac{\beta}{T^{\beta}} \int_{0}^{T}(T-s)^{\beta-1} x(s) d s \\
& +\frac{\beta}{T^{\beta}} \int_{0}^{T} G\left(t_{0}, s\right)(T-s)^{\beta-1} g\left(s, x(s), D_{0+}^{\alpha} x(s)\right) d s \\
\geq & \frac{\beta}{T^{\beta}} \int_{0}^{T}(T-s)^{\beta-1} m\|x\| d s \\
& +\frac{\beta}{T^{\beta}} \int_{0}^{T} G\left(t_{0}, s\right)(T-s)^{\beta-1} g\left(s, x(s), D_{0+}^{\alpha} x(s)\right) d s \\
\geq & m\|x\|+\frac{\beta}{T^{\beta}} \int_{0}^{T} G\left(t_{0}, s\right)(T-s)^{\beta-1}\left[h_{1}(x(s))+h_{2}\left(D_{0+}^{\alpha} x(s)\right)\right] d s \\
= & m\|x\|+\frac{\beta}{T^{\beta}} \int_{0}^{T} G\left(t_{0}, s\right)(T-s)^{\beta-1}\left[\frac{h_{1}(x(s))}{x(s)} \cdot x(s)+\frac{h_{2}\left(D_{0+}^{\alpha} x(s)\right)}{D_{0+}^{\alpha} x(s)} \cdot D_{0+}^{\alpha} x(s)\right] d s \\
\geq & m\|x\|+\frac{\beta}{T^{\beta}} \int_{0}^{T} G\left(t_{0}, s\right)(T-s)^{\beta-1}\left[\frac{h_{1}(x(s))}{x(s)}+\frac{h_{2}\left(D_{0+}^{\alpha} x(s)\right)}{D_{0+}^{\alpha} x(s)}\right] \cdot m\|x\| d s \\
\geq & m\|x\|+m\|x\| \cdot \frac{\beta}{T^{\beta}} \int_{0}^{T} G\left(t_{0}, s\right)(T-s)^{\beta-1}\left[\frac{h_{1}(r)}{r}+\frac{h_{2}(r)}{r}\right] d s \\
\geq & m\|x\|+m\|x\| \cdot \frac{1-m}{m} \\
= & \|x\| .
\end{aligned}
$$

To conclude, for all $x \in C_{1}\left(x_{0}\right) \cap \partial \Omega_{1}$, we have $\|x\| \leq \sigma\left(x_{0}\right)\|\Psi x\|_{\infty} \leq \sigma\left(x_{0}\right)\|\Psi x\|$, i.e., (A6) holds. For $x \in \partial \Omega_{2}$, (Hb2) implies

$$
\begin{aligned}
& {\left[\left(P_{1}+J Q_{1} N_{1}\right) \circ \gamma\right] x(t)} \\
& \quad=P_{1}(|x(t)|)+J Q_{1} N_{1}(|x(t)|)
\end{aligned}
$$




$$
\begin{aligned}
& =\frac{\beta}{T^{\beta}} \int_{0}^{T}(T-s)^{\beta-1}|x(s)| d s+\frac{\beta}{T^{\beta}} \int_{0}^{T}(T-s)^{\beta-1} g\left(s,|x(s)|, D_{0+}^{\alpha}|x(s)|\right) d s \\
& \geq \frac{\beta}{T^{\beta}} \int_{0}^{T}(T-s)^{\beta-1}(1-\kappa)|x(s)| d s \\
& \geq 0 .
\end{aligned}
$$

Thus, for $x \in \partial \Omega_{2}$, one has $\left[\left(P_{1}+J Q_{1} N_{1}\right) \circ \gamma\right] x(t) \subset C_{1}$. Then (A7) holds.

Finally, we prove (A8). For $x(t) \in \bar{\Omega}_{2} \backslash \Omega_{1}$, based on (H2) and (33), we have

$$
\begin{aligned}
\Psi_{\gamma} x(t)= & {\left[\left(P_{1}+J Q_{1} N_{1}+K_{P}\left(I-Q_{1}\right) N_{1}\right) \circ \gamma\right] x(t) } \\
= & \left(P_{1}+J Q_{1} N_{1}+K_{P}\left(I-Q_{1}\right) N_{1}\right)|x(t)| \\
= & P_{1}(|x(t)|)+\left[J Q_{1} N_{1}+K_{P}\left(I-Q_{1}\right) N_{1}\right]|x(t)| \\
= & \frac{\beta}{T^{\beta}} \int_{0}^{T}(T-s)^{\beta-1}|x(s)| d s \\
& +\frac{\beta}{T^{\beta}} \int_{0}^{T} G(t, s)(T-s)^{\beta-1} g\left(s,|x(s)|, D_{0+}^{\alpha}|x(s)|\right) d s \\
\geq & \frac{\beta}{T^{\beta}} \int_{0}^{T}(T-s)^{\beta-1}|x(s)| d s+\frac{\beta}{T^{\beta}} \int_{0}^{T} G(t, s)(T-s)^{\beta-1}(-\kappa|x(s)|) d s \\
= & \frac{\beta}{T^{\beta}} \int_{0}^{T}(T-s)^{\beta-1}|x(s)|(1-\kappa G(t, s)) d s \\
\geq & 0 .
\end{aligned}
$$

Hence, $\Psi_{\gamma}\left(\bar{\Omega}_{2} \backslash \Omega_{1}\right) \subset C_{1}$, that is, (A8) holds. Hence, applying Theorem 2, PBVP (3) has a positive solution $x^{*}(t)$ on $[0, T]$ with $r \leq\left\|x^{*}(t)\right\| \leq B$. This completes the proof.

\section{Examples}

In this section, two examples will be given to illustrate our main result.

Example 1 Consider the following PBVP for the nonlinear fractional differential equation:

$$
\left\{\begin{array}{l}
D_{0^{+}}^{\frac{3}{4}}\left(\left(t^{2}-t+\frac{5}{4}\right) D_{0^{+}}^{\frac{1}{2}} x(t)\right)=-\frac{8}{3}+\frac{1}{24} x^{2}(t)+t e^{-\left(D_{0^{+}}^{\frac{1}{2}} x(t)\right)^{2}}, \quad t \in[0,1], \\
x(0)=x(1), \quad D_{0^{+}}^{\frac{1}{2}} x(0)=D_{0^{+}}^{\frac{1}{2}} x(1) .
\end{array}\right.
$$

According to PBVP (1), we get that $p(t)=t^{2}-t+\frac{5}{4}, M=1, \alpha=\frac{3}{4}, \beta=\frac{1}{2}, T=1$, and

$$
f(t, u, v)=-\frac{8}{3}+\frac{1}{24} u^{2}+t e^{-v^{2}} .
$$

Let $a(t)=4, b(t)=\frac{1}{24}, c(t)=0, B=8$. A simple calculation shows that $\|b\|_{\infty}=\frac{1}{24},\|c\|_{\infty}=0$, and

$$
\begin{aligned}
& u f(t, u, v)=u\left(\frac{u^{2}-64}{24}+t e^{-v^{2}}\right)>0 \quad(\text { or }<0), \forall t \in[0,1], v \in \mathbb{R},|u|>8, \\
& \gamma=\frac{2}{\Gamma\left(\frac{3}{4}+1\right)}\left(\frac{2 \cdot \frac{1}{24}}{\Gamma\left(\frac{1}{2}+1\right)}+0\right)=0.2046<1 .
\end{aligned}
$$


All assumptions of Theorem 1 are satisfied. Hence PBVP (46) admits at least one solution.

Example 2 Consider the fractional periodic boundary value problem

$$
\begin{cases}D_{0^{+}}^{0.5} D_{0^{+}}^{1.5} x(t)=g\left(t, x(t), D_{0^{+}}^{1.5} x(t)\right), & t \in[0,1] \\ x(0)=x(1), & x^{\prime}(0)=x^{\prime}(1),\end{cases}
$$

where $g\left(t, x, D_{0^{+}}^{1.5} x\right)=\frac{2}{5}\left(1+t^{2}\right)\left(-\frac{1}{2} x-\frac{1}{2} D_{0^{+}}^{1.5} x+\frac{5}{4}\right)$.

Corresponding to PBVP (47), we have that $\beta=0.5, \alpha=1.5, T=1$, and

$$
G(t, s)=\left\{\begin{array}{l}
-\frac{4(1-s)^{1.5}}{3}-\frac{7 s}{3}+2 t s+\frac{7 t}{6}-\frac{1}{2} t^{2}-\frac{2}{3} t^{1.5}+\frac{\Gamma(1.5) \Gamma(1.5)}{2}+\frac{20}{9}, \\
\quad 0 \leq s<t \leq 1, \\
-\frac{4(1-s)^{1.5}}{3}-\frac{s}{3}+2 t s-\frac{5 t}{6}-\frac{1}{2} t^{2}-\frac{2}{3} t^{1.5}+\frac{\Gamma(1.5) \Gamma(1.5)}{2}+\frac{20}{9}, \\
0 \leq t<s \leq 1 .
\end{array}\right.
$$

By a simple calculation, we obtain $G(t, s)<2.5$. Hence, we take $\kappa=\frac{2}{5}$ based on (33). In addition, we find that if $t \in[0,1], x \in[0,60]$, and $D_{0+}^{1.5} x \in[0,60]$, the following inequality holds:

$$
\begin{aligned}
& -2 x(t)-2 D_{0+}^{1.5} x(t) \leq g\left(t, x(t), D_{0+}^{1.5} x(t)\right) \leq-\frac{1}{5} x(t)-\frac{1}{5} D_{0+}^{1.5} x(t)+1, \\
& g\left(t, x(t), D_{0+}^{1.5} x(t)\right) \leq-\left|g\left(t, x(t), D_{0+}^{1.5} x(t)\right)\right|+\frac{6}{5} x(t)+\frac{6}{5} D_{0+}^{1.5} x(t)+1 .
\end{aligned}
$$

So we can choose $B=60, c_{1}=c_{2}=\frac{1}{5}, c_{3}=1, b_{1}=1, b_{2}=b_{3}=\frac{6}{5}, b_{4}=1$.

Furthermore, it is easy to verify that

$$
\begin{aligned}
& \Gamma(3-\alpha) \Gamma(\alpha+\beta)-2 \kappa(\alpha-1) T^{\alpha+2 \beta-2}=0.4862>0, \\
& b_{1} c_{1} c_{2} \beta+b_{1} c_{1}^{2} \beta+8 T^{\alpha+\beta-1} b_{2} c_{2}^{2}-8 T^{\alpha+\beta-1} b_{3} c_{1} c_{2}=\frac{1}{25}>0, \\
& A_{1}=58.5, \quad A_{2}=3.085, \quad \frac{c_{3}}{c_{1}}=5 \quad \text { and } \quad B=60>\max \left\{A_{1}, A_{2}, \frac{c_{3}}{c_{1}}\right\} .
\end{aligned}
$$

Therefore, $(\mathrm{Hb} 1)$ is satisfied.

We take $r=0.5 \in[0,60], h_{1}(x)=\frac{x}{10}, h_{2}\left(D_{0+}^{1.5} x\right)=\frac{D_{0+}^{1.5} x}{10}$. By calculation, we obtain

$$
\begin{aligned}
g\left(t, x(t), D_{0+}^{1.5} x(t)\right) & \geq h_{1}(x)+h_{2}\left(D_{0+}^{1.5} x\right) \\
& =\frac{x(t)}{10}+\frac{D_{0+}^{1.5} x(t)}{10}, \quad\left(t, x, D_{0+}^{1.5} x\right) \in[0,1] \times(0,0.5]^{2}
\end{aligned}
$$

and

$$
\frac{h_{1}(x)}{x}=\frac{h_{2}\left(D_{0+}^{1.5} x\right)}{D_{0+}^{1.5} x}=\frac{1}{10},
$$

which is nonincreasing on $(0,0.5]$. 
Let $t_{0}=0$, then we have

$$
G\left(t_{0}, s\right)=G(0, s)=-\frac{4(1-s)^{1.5}}{3}+\frac{4(1-s)}{3}+1.2816>1.2816>0
$$

Using the given data, we have

$$
\frac{\beta}{T^{\beta}} \frac{h_{i}(r)}{r} \int_{0}^{1} G(0, s)(1-s)^{\beta-1} d s \approx 0.1391 \geq \frac{1-m}{2 m}, \quad i=1,2,
$$

holds for $m=0.8$. One sees that ( $\mathrm{Hb} 2)$ is satisfied. In consequence, the conclusion of Theorem 3 implies that problem (47) has a positive solution on $[0,1]$.

\section{Conclusion}

We have proved the existence of solutions for two classes of fractional differential equations with periodic boundary value conditions, where certain nonlinear growth conditions of the nonlinearity need to be satisfied. The problem is issued by applying the LeggettWilliams norm-type theorem for coincidences. We also provide examples to make our results clear.

Acknowledgements

Not applicable.

Funding

This work is partially supported by the National Science Foundation of China (51476047, U1637208) and the Natural Scientific Foundation of Heilongjiang Province in China (A2016003).

Abbreviations

PBVP, Periodic boundary value problems..

Availability of data and materials

Not applicable.

Competing interests

The authors declare that they have no competing interests.

\section{Authors' contributions}

The authors read and approved the final manuscript.

\section{Publisher's Note}

Springer Nature remains neutral with regard to jurisdictional claims in published maps and institutional affiliations.

Received: 17 January 2018 Accepted: 5 November 2018 Published online: 15 November 2018

\section{References}

1. Baleanu, D., Diethelm, K., Scalas, E., Trujillo, J.: Fractional Calculus Models and Numerical Methods. Series on Complexity, Nonlinearity and Chaos. World Scientific, Boston (2012)

2. Magin, R.L.: Fractional Calculus in Bioengineering. Begell House, Connecticut (2006)

3. Mainardi, F.: Fractional Calculus and Waves in Linear Viscoelasticity: An Introduction to Mathematical Models. Imperial College Press, London (2010)

4. Tenreiro Machado, A., Kiryakova, V., Mainardi, F.: Recent history of fractional calculus. Commun. Nonlinear Sci. Numer. Simul. 16, 1140-1153 (2011)

5. Carpinteri, A., Mainardi, F.: Fractals and Fractional Calculus in Continuum Mechanics. Springer, Vienna (1997)

6. Podlubny, I.: Fractional Differential Equation. Academic Press, San Diego (1999)

7. Kilbas, A., Srivastava, H., Trujillo, J.: Theory and Applications of Fractional Differential Equations. Elsevier, Amsterdam (2006)

8. Baleanu, D. Tenreiro Machado, J., Luo, A.: Fractional Dynamics and Control. Springer, New York (2012)

9. Bai, Z:: On positive solutions of a nonlocal fractional boundary value problem. Nonlinear Anal. 72(2), 916-924 (2010)

10. Ahmad, B.: Existence of solutions for irregular boundary value problems of nonlinear fractional differential equations. Appl. Math. Lett. 23(4), 390-394 (2010) 
11. Graef, J., Kong, L., Yang, B.: Positive solutions for a fractional boundary value problem. Appl. Math. Lett. 56, 49-55 (2016)

12. Szekeres, B., Izsák, F: Finite element approximation of fractional order elliptic boundary value problems. J. Comput. Appl. Math. 292, 553-561 (2016)

13. Graef, J., Kong, L., Kong, Q.: Application of the mixed monotone operator method to fractional boundary value problems. Fract. Differ. Calc. 2, 87-98 (2012)

14. Khan, R., Shah, K.: Existence and uniqueness of solutions to fractional order multi-point boundary value problems. Commun. Appl. Anal. 19, 515-526 (2015)

15. Al-Mdallal, Q., Hajji, M.: A convergent algorithm for solving higher-order nonlinear fractional boundary value problems. Fract. Calc. Appl. Anal. 18(6), 1423-1440 (2015)

16. Ahmad, B., Nieto, J.: Anti-periodic fractional boundary value problems with nonlinear term depending on lower order derivative. Fract. Calc. Appl. Anal. 15(3), 451-462 (2012)

17. Agarwal, R., Ahmad, B.: Existence theory for anti-periodic boundary value problems of fractional differential equations and inclusions. Comput. Math. Appl. 62, 1200-1214 (2011)

18. Benchohra, M., Hamidi, N., Henderson, J.: Fractional differential equations with anti-periodic boundary conditions. Numer. Funct. Anal. Optim. 34(4), 404-414 (2013)

19. Shah, K., Khan, R.: Existence and uniqueness of positive solutions to a coupled system of nonlinear fractional order differential equations with anti periodic boundary conditions. Differ. Equ. Appl. 7(2), 243-262 (2015)

20. Ahmad, B., Nieto, J.: Anti-periodic fractional boundary value problems. Comput. Math. Appl. 62(3), 1150-1156 (2011)

21. Li, X., Han, Z., Sun, S.: Anti-periodic boundary value problems for fractional q-difference equations. J. Appl. Math. Comput. 50(1-2), 243-257 (2016)

22. Zhang, Y.: The existence of solutions to nonlinear second order periodic boundary value problems. Nonlinear Anal. 76, 140-152 (2013)

23. Liu, J., Feng, H.: Positive solutions of periodic boundary value problems for second-order differential equations with the nonlinearity dependent on the derivative. J. Appl. Math. Comput. 49(1-2), 343-355 (2015)

24. Liu, X., Zhang, Y., Shi, H., Deng, X.: Periodic and subharmonic solutions for fourth-order nonlinear difference equations. Appl. Math. Comput. 236, 613-620 (2014)

25. Liu, B., Liu, L., Wu, Y.: Existence of nontrivial periodic solutions for a nonlinear second order periodic boundary value problem. Nonlinear Anal. 72(7-8), 3337-3345 (2010)

26. Chen, C., Liu, W.: Solvability of periodic boundary-value problems for second-order nonlinear differential equation involving fractional derivatives. Electron. J. Differ. Equ. 2014, 261, 1-9 (2014)

27. $\mathrm{Hu}, \mathrm{L}$., Zhang, S.: Existence of positive solutions to a periodic boundary value problems for nonlinear fractional differential equations at resonance. J. Fract. Calc. Appl. 8(2), 19-31 (2017)

28. Mawhin, J.: Topological Degree Methods in Nonlinear Boundary Value Problems. CBMS Conf. Series, vol. 40. Am. Math. Soc., Providence (1979)

29. Chen, T., Liu, W., Liu, J.: Solvability of periodic boundary value problem for fractional $p$-Laplacian equation. Appl. Math. Comput. 244, 422-431 (2014)

30. O'Regan, D., Zima, M.: Leggett-Williams norm-type theorems for coincidences. Arch. Math. 87, 233-244 (2006)

\section{Submit your manuscript to a SpringerOpen ${ }^{\circ}$ journal and benefit from:}

- Convenient online submission

- Rigorous peer review

- Open access: articles freely available online

- High visibility within the field

- Retaining the copyright to your article

Submit your next manuscript at $\gg$ springeropen.com 